\title{
Transport properties of quasi-free Fermions
}

\author{
W. Aschbacher ${ }^{1}$, V. Jakšić ${ }^{2}$, Y. Pautrat ${ }^{3}$, C.-A. Pillet ${ }^{4}$ \\ ${ }^{1}$ Technische Universität München \\ Zentrum Mathematik M5 \\ D-85747 Garching, Germany \\ ${ }^{2}$ Department of Mathematics and Statistics \\ McGill University \\ 805 Sherbrooke Street West \\ Montreal, QC, H3A 2K6, Canada \\ ${ }^{3}$ Laboratoire de Mathématiques \\ Université Paris-Sud \\ 91405 Orsay cedex, France \\ ${ }^{4}$ CPT-CNRS Université du Sud Toulon-Var \\ B.P. 20132 \\ 83957 La Garde cedex, France
}

October 23, 2006

\begin{abstract}
Using the scattering approach to the construction of Non-Equilibrium Steady States proposed by Ruelle we study the transport properties of systems of independent electrons. We show that Landauer-Büttiker and Green-Kubo formulas hold under very general conditions.
\end{abstract}




\section{Introduction}

Recently, there has been a renewed interest in mathematical aspects of nonequilibrium quantum statistical mechanics. The general definition of Non-Equilibrium Steady State (NESS) proposed by Ruelle in [Ru1], [Ru2] has been at the center of most of the resulting works. Paradigmatic examples of NESS are given by open quantum systems driven out of equilibrium by thermodynamical forces. In these examples a "small system" $\mathcal{S}$, with a finite number of degrees of freedom, interacts with several extended ideal reservoirs $\mathcal{R}_{1}, \ldots, \mathcal{R}_{M}$. At time $t=0$ these reservoirs are in thermal equilibrium at inverse temperatures $\beta_{1}, \ldots, \beta_{M}{ }^{1}$ If these parameters do not all take the same value then, as $t \rightarrow \infty$, the coupled system $\mathcal{S}+\mathcal{R}_{1}+\cdots+\mathcal{R}_{M}$ approaches a steady state which carries non-trivial currents. To our knowledge two methods are currently available for the construction of NESS: the $C^{*}$-scattering approach proposed by Ruelle in [Ru1] (see also [Ro] for the foundational work on $C^{*}$-scattering theory) and the spectral approach developed in [JP1].

Starting decades before these new developments with the pioneering work of Landauer [L], large efforts have been (and still are) invested in the elaboration of a theory of coherent electronic transport in mesoscopic systems (see [I] or [D]). However, despite the obvious relation between this theory and open systems, the mathematical status of the former remained obscure.

In this paper we consider coherent electronic transport in the independent electrons approximation. Our aim is to show that the Landauer-Büttiker formalism used in the physics literature to deal with this class of models is a special instance of Ruelle's scattering approach. More specifically we shall use this approach to obtain an explicit formula for the NESS. Using this formula we shall derive the Landauer-Büttiker formula which expresses the steady currents flowing through a sample in terms of the scattering data of this sample. We shall also discuss the linear response theory of independent electrons models.

The above relation between the Landauer-Büttiker formalism and Ruelle's approach has already been noticed in specific models where the scattering matrix can be computed explicitly (see [TT1], [AJPP], [TT2], [TT3], and [AH], [AP] for related results). In this paper we shall use the stationary approach to Hilbert space scattering to deal with more general models. In $[\mathrm{BP}]$ we will follow a time-dependent approach to this problem, based on the Mourre estimate.

Similar results have been obtained in [AEG] for the current in a quantum pump in the adiabatic limit. We also note that a derivation of the Landauer-Büttiker formula from microscopic dynamics has been proposed in [CJM]. However, in our perspective, this last result is unsatisfactory for at least two reasons. Firstly, it only deals with conductivity (and not with the currents). Secondly, and more importantly, it does not make direct connection with NESS and non-equilibrium statistical mechanics.

\footnotetext{
${ }^{1}$ Depending on the nature of these reservoirs, other intensive thermodynamic parameters (e.g., chemical potentials $\left.\mu_{1}, \ldots, \mu_{M}\right)$ can be specified.
} 
This paper is organized as follows. In Section 2 we briefly review some basic facts about $C^{*}$-dynamical systems, canonical anticommutation relations and quasi-free states of Fermionic systems. In Section 3 we introduce the model and discuss its NESS and flux observables. Section 4 contains our main results: the Landauer-Büttiker formulas for the steady currents and the corresponding linear response formulas. Section 5 is a complement to the recent series of papers [J0P1]-[J0P4] on linear response to thermodynamical forces. There we discuss fluctuation-dissipation (Green-Kubo) formulas. Finally Section 6 is devoted to the proofs of our main results.

The fluctuation theory of the independent electrons models concerns Central Limit Theorem and Large Deviation Principle for the steady state currents. For reasons of space we shall discuss these topics in the continuation of this paper.

This paper is based on parts of a series of lectures given by the fourth author at the Simion Stoilow Institute of Mathematics in Bucharest in June 2004/January 2005 and at the Erwin Schrödinger Institute for Theoretical Physics in Vienna (ESI) during the spring 2005. Other parts of the material covered by these lectures have been published elsewhere ([JP2], [AJPP], [JKP]).

Acknowledgments. Part of this work has been done during the stay of Y.P. and the visit of C.-A. P. at CRM and McGill University in Montreal. Other parts where done during the stays of W. A. and V. J. at CPT-Marseille. C.-A. P. thanks Jan Dereziński and Gian Michele Graf for the invitation to give a series of lecture during the ESI workshop "Open Quantum Systems" and for helpful discussions; he also thanks Radu Purice for the invitations at the Simion Stoilow Institute of Mathematics in Bucharest. Finally he is grateful to Leonid Pastur for useful discussions. The research of V.J. was partly supported by NSERC.

\section{Quasi-Free Fermions}

For notational purposes in this section we briefly review the mathematical framework used for the description of an infinitely extended ideal Fermi gas at positive density. For references and additional information we refer the reader to [BR, AJPP].

\section{$2.1 \quad C^{*}$-Dynamical Systems}

A $C^{*}$-dynamical system is a pair $(\mathcal{O}, \tau)$ where $\mathcal{O}$ is a $C^{*}$-algebra and $t \mapsto \tau^{t}$ a strongly continuous group of $*$-automorphisms of $\mathcal{O}$. A state on $\mathcal{O}$ is a normalized positive linear functional $\omega: \mathcal{O} \rightarrow \mathbb{C}$. A state $\omega$ is $\tau$-invariant if $\omega \circ \tau^{t}=\omega$ holds for all $t \in \mathbb{R}$.

The GNS construction associates to any state $\omega$ a cyclic representation $\left(\mathcal{H}_{\omega}, \pi_{\omega}, \Omega_{\omega}\right)$ of $\mathcal{O}$. $\mathcal{H}_{\omega}$ is a Hilbert space, $\pi_{\omega}: \mathcal{O} \rightarrow \mathcal{B}\left(\mathcal{H}_{\omega}\right)$ a $*$-morphism and $\Omega_{\omega} \in \mathcal{H}_{\omega}$ a unit vector such that

$$
\omega(A)=\left(\Omega_{\omega}, \pi_{\omega}(A) \Omega_{\omega}\right)
$$


for all $A \in \mathcal{O}$ and

$$
\overline{\pi_{\omega}(\mathcal{O}) \Omega_{\omega}}=\mathcal{H}_{\omega}
$$

A state $\eta$ is $\omega$-normal if there exists a density matrix $\rho$ on $\mathcal{H}_{\omega}$ such that

$$
\eta(A)=\operatorname{Tr}\left(\rho \pi_{\omega}(A)\right),
$$

for all $A \in \mathcal{O}$.

A state $\omega$ is ergodic (resp. mixing) for the $C^{*}$-dynamical system $(\mathcal{O}, \tau)$ if, for any $\omega$-normal state $\eta$ and any $A \in \mathcal{O}$ one has

$$
\lim _{T \rightarrow \infty} \frac{1}{T} \int_{0}^{T} \eta \circ \tau^{t}(A) \mathrm{d} t=\omega(A), \quad\left(\text { resp. } \lim _{t \rightarrow \infty} \eta \circ \tau^{t}(A)=\omega(A)\right) .
$$

Thermal equilibrium states of the $C^{*}$-dynamical system $(\mathcal{O}, \tau)$ are characterized by $\beta$ KMS condition, where $\beta>0$ is the inverse temperature. We recall that $\omega$ is a $(\tau, \beta)$-KMS state if, for any $A, B \in \mathcal{O}$, the correlation function $t \mapsto F_{A B}(t)=\omega\left(A \tau^{t}(B)\right)$ extends to a function which is analytic on the $\operatorname{strip} S_{\beta}=\{z \in \mathbb{C} \mid 0<\operatorname{Im}(z)<\beta\}$, continuous on its closure and satisfies $F_{A B}(t+\mathrm{i} \beta)=\omega\left(\tau^{t}(B) A\right)$.

\subsection{CAR-Algebras and Quasi-Free States}

Let $\mathfrak{h}$ and $h$ be the quantum mechanical Hilbert space and the Hamiltonian of a single Fermion. We shall always assume that $\mathfrak{h}$ is separable. The Fermionic Fock space over $\mathfrak{h}$ is denoted by $\Gamma_{-}(\mathfrak{h})$ and, for $f \in \mathfrak{h}, a(f)$ and $a^{*}(f)$ denote the corresponding Fermionic annihilation and creation operators. Recall that these are bounded operators on $\Gamma_{-}(\mathfrak{h})$, of norm $\|f\|$, satisfying the canonical anticommutation relations (CAR)

$$
\left\{a(f), a^{*}(g)\right\}=a(f) a^{*}(g)+a^{*}(g) a(f)=(f, g) .
$$

In the sequel $a^{\#}$ denotes either $a$ or $a^{*}$ and $\varphi(f)=2^{-1 / 2}\left(a(f)+a^{*}(f)\right)$ denotes the field operator. The Fermi algebra $\operatorname{CAR}(\mathfrak{h})$ is the $C^{*}$-algebra generated by the set $\{\varphi(f) \mid f \in$ $\mathfrak{h}\}$. If $c$ is a trace class operator on $\mathfrak{h}$, then $\mathrm{d} \Gamma(c) \in \mathrm{CAR}(\mathfrak{h})$ and one has

$$
\|\mathrm{d} \Gamma(c)\| \leq\|c\|_{1}
$$

The Hamiltonian $H=\mathrm{d} \Gamma(h)$ and the Fermion number operator $N=\mathrm{d} \Gamma(I)$ define two commuting, strongly continuous groups of $*$-automorphisms of $\mathrm{CAR}(\mathfrak{h})$ : the dynamical group

$$
\tau^{t}(A) \equiv \mathrm{e}^{i t H} A \mathrm{e}^{-i t H},
$$

and the gauge group

$$
\vartheta^{s}(A) \equiv \mathrm{e}^{i s N} A \mathrm{e}^{-i s N} .
$$


They coincide with the groups of Bogoliubov automorphisms characterized by

$$
\tau^{t}\left(a^{\#}(f)\right)=a^{\#}\left(\mathrm{e}^{i t h} f\right), \quad \vartheta^{s}\left(a^{\#}(f)\right)=a^{\#}\left(\mathrm{e}^{i s} f\right) .
$$

Thus, to the pair $(\mathfrak{h}, h)$ which characterizes the one-body dynamics we can associate the $C^{*}$-dynamical system $\left(\mathrm{CAR}(\mathfrak{h}), \tau^{t}\right)$ which describes the corresponding ideal Fermi gas. Physical observables are gauge-invariant and they are elements of the $\tau^{t}$-invariant $C^{*}$ subalgebra

$$
\mathrm{CAR}_{\vartheta}(\mathfrak{h})=\left\{A \in \mathrm{CAR}(\mathfrak{h}) \mid \vartheta^{s}(A)=A \text { for all } s \in \mathbb{R}\right\} .
$$

Note that if $\mathfrak{K}$ is a subspace of $\mathfrak{h}$ then, $\operatorname{CAR}_{\vartheta}(\mathfrak{K})$ is a subalgebra of $\operatorname{CAR}_{\vartheta}(\mathfrak{h})$.

If $\omega$ is a state on $\operatorname{CAR}(\mathfrak{h})$, then there exists a self-adjoint operator $\varrho$ on $\mathfrak{h}$ such that $0 \leq \varrho \leq I$ and

$$
\omega\left(a^{*}(g) a(f)\right)=(f, \varrho g) .
$$

We call $\varrho$ the density operator of $\omega$. Note that if $c$ is a trace class operator on $\mathfrak{h}$ then

$$
\omega(\mathrm{d} \Gamma(c))=\operatorname{Tr}(\varrho c) .
$$

Reciprocally, to any self-adjoint operator $\varrho$ on $\mathfrak{h}$ such that $0 \leq \varrho \leq I$, one can associate a state $\omega_{\varrho}$ on $\operatorname{CAR}(\mathfrak{h})$ which is completely characterized by the fact that

$$
\omega_{\varrho}\left(a^{*}\left(g_{n}\right) \cdots a^{*}\left(g_{1}\right) a\left(f_{1}\right) \cdots a\left(f_{m}\right)\right)=\delta_{n m} \operatorname{det}\left\{\left(f_{i}, \varrho g_{j}\right)\right\} .
$$

This state is invariant under the action of the gauge group $\vartheta$ and satisfies the Fermionic Wick decomposition rule

$$
\omega_{\varrho}\left(\varphi\left(f_{1}\right) \cdots \varphi\left(f_{2 n}\right)\right)=\sum_{\pi \in \mathcal{P}_{n}} \prod_{j=1}^{n} \omega_{\varrho}\left(\varphi\left(f_{\pi(2 j-1)}\right) \varphi\left(f_{\pi(2 j)}\right)\right)
$$

where the sum runs over the set $\mathcal{P}_{n}$ of all permutations $\pi$ of the set $\{1, \cdots, 2 n\}$ such that $\pi(2 j-1)<\pi(2 j)$ and $\pi(2 j-1)<\pi(2 j+1)$. The state $\omega_{\varrho}$ is called the gauge-invariant quasi-free state of density $\varrho$.

Under the time evolution $\tau$, gauge invariant quasi-free states transform into gauge invariant quasi-free states, i.e.,

$$
\omega_{\varrho} \circ \tau^{t}=\omega_{\varrho},
$$

where $\varrho_{t}=\mathrm{e}^{-\mathrm{i} t h} \varrho \mathrm{e}^{\mathrm{i} t h}$. In particular, the state $\omega_{\varrho}$ is $\tau$-invariant if and only if $\varrho$ commutes with the Hamiltonian $h$.

For any $\beta>0$ and $\mu \in \mathbb{R}$ we denote by $\omega_{\beta \mu}$ the gauge-invariant quasi-free state with density $f_{\beta \mu}(h)$ where $f_{\beta \mu}(\varepsilon)=\left(1+\mathrm{e}^{\beta(\varepsilon-\mu)}\right)^{-1}$ is the Fermi-Dirac distribution at inverse temperature $\beta$ and chemical potential $\mu$. This state is the unique $\beta$-KMS state on $\operatorname{CAR}(\mathfrak{h})$ for the dynamics $\tau^{t} \circ \vartheta^{-\mu t}$. Note that $\tau^{t} \circ \vartheta^{-\mu t}$ coincide with $\tau^{t}$ on $\operatorname{CAR}_{\vartheta}(\mathfrak{h})$. Any $(\tau, \beta)$ KMS state on $\mathrm{CAR}_{\vartheta}(\mathfrak{h})$ extends uniquely to a state of the form $\omega_{\beta \mu}$ for some $\mu \in \mathbb{R}$. The 


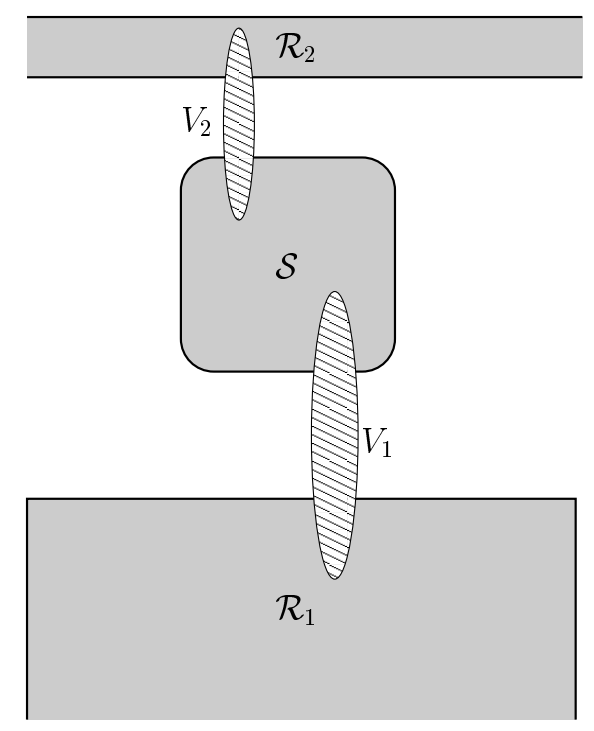

Figure 1: EBB connected to two reservoirs.

$C^{*}$-dynamical system $\left(\mathrm{CAR}_{\vartheta}(\mathfrak{h}), \tau^{t}\right)$ equipped with the state $\omega_{\beta \mu}$ describes a free Fermi gas at thermal equilibrium, in the grand canonical ensemble at inverse temperature $\beta$ and chemical potential $\mu$.

A gauge-invariant quasi-free state $\omega_{\varrho}$ is ergodic for the ideal Fermi gas $\left(\mathrm{CAR}(\mathfrak{h}), \tau^{t}\right)$ if and only if $h$ has empty point spectrum. If $h$ has empty singular spectrum, then $\omega_{\varrho}$ is mixing.

\section{The Electronic Black-Box (EBB) Model}

We call electronic black-box a free Fermi gas consisting of a spatially confined region $\mathcal{S}$ - the sample - coupled to several spatially extended reservoirs $\mathcal{R}_{1}, \ldots, \mathcal{R}_{M}$ (see Figure 1). Fermions can flow between the reservoir $\mathcal{R}_{k}$ and the sample $\mathcal{S}$ through a junction $V_{k}$. We denote by $\mathfrak{h}_{\mathcal{S}}$ the one-particle Hilbert space of the sample and by $h_{\mathcal{S}}$ its one-particle Hamiltonian. Each reservoir $\mathcal{R}_{k}$ is likewise described by a one-particle Hilbert space $\mathfrak{h}_{k}$ and a one-particle Hamiltonian $h_{k}$. The complete one-particle Hilbert space of the system is

$$
\mathfrak{h}=\mathfrak{h}_{\mathcal{S}} \oplus \mathfrak{h}_{\mathcal{R}}, \quad \mathfrak{h}_{\mathcal{R}}=\bigoplus_{k=1}^{M} \mathfrak{h}_{k},
$$

and the one-particle Hamiltonian of the decoupled system is

$$
h_{0}=h_{\mathcal{S}} \oplus h_{\mathcal{R}}, \quad h_{\mathcal{R}}=\bigoplus_{k=1}^{M} h_{k} .
$$


Let $h$ be the one-particle Hamiltonian of the coupled system. We denote by $\tau_{0}^{t}\left(\right.$ resp. $\left.\tau^{t}\right)$ the Bogoliubov dynamics on $\mathcal{O}=\mathrm{CAR}(\mathfrak{h})$ generated by $h_{0}$ (resp. $h$ ). The EBB model (the coupled joint system $\mathcal{S}+\mathcal{R})$ is described by the $C^{*}$-dynamical system $\left(\mathcal{O}, \tau^{t}\right)$. The simplest non-trivial version of this model (the Simple Electronic Black-Box (SEBB)) has been studied in the lecture notes [AJPP] and [JKP]. The purpose of this paper is to extend this analysis to a larger class of models.

We shall assume that both $h_{0}$ and $h$ are bounded below.

(H1) $h_{0}+E_{0} \geq 0$ and $h+E_{0} \geq 0$ for some $E_{0} \in \mathbb{R}$.

Concerning the junction we shall make the following regularity assumption

(H2) For some integer $p>0$ or $p=-1$ the difference

$$
\left(1+h+E_{0}\right)^{-p}-\left(1+h_{0}+E_{0}\right)^{-p},
$$

is trace class.

The case $p=-1$ is particular since then $\mathrm{d} \Gamma\left(h-h_{0}\right) \in \mathcal{O}$ which implies that the coupled dynamics $\tau^{t}$ is a local perturbation of the decoupled one $\tau_{0}^{t}$. From a mathematical point of view, the theory of local perturbations of $C^{*}$-dynamical systems is very natural and well developed. Most of the rigorous results in nonequilibrium statistical mechanics of open quantum systems deal with such local perturbations. However, we expect more general perturbations corresponding to $p>0$ to play an important role, especially in the high temperature regime.

Denote by $\rho(A)$ the resolvent set of an operator $A$. The following simple consequence of Assumptions (H1)-(H2) is proved in Subsection 6.1.

Lemma 3.1 Under Assumptions (H1)-(H2),

$$
(h-z)^{-q}-\left(h_{0}-z\right)^{-q}
$$

is trace class for any integer $q \geq p$ and any $z \in \rho\left(h_{0}\right) \cap \rho(h)$. Moreover, if $\varphi \in C_{0}^{\infty}(\mathbb{R})$ then

$$
\varphi\left(\left(1+h+E_{0}\right)^{-p}\right)-\varphi\left(\left(1+h_{0}+E_{0}\right)^{-p}\right)
$$

is trace class.

We shall also assume:

(H3) $h$ has empty singular continuous spectrum. 
To the best of our knowledge, the steady state currents of independent electrons models in presence of singular continuous spectra have not been studied in the literature. We remark that if (H2) holds with $p=-1$, then in a suitable probabilistic setup Assumption (H3) is generically satisfied, see [JL1], [JL2].

Most of our results require Assumptions (H1)-(H3). Occasionally we will invoke the Time Reversal Invariance Assumption:

(TRI) There exists a conjugation $J$ on $\mathfrak{h}$ such that

$$
J h_{0}=h_{0} J, \quad J h=h J
$$

Under Assumption (TRI) the map $j\left(a^{\#}(f)\right)=a^{\#}(J f)$ extends to a skew *-automorphism of $\mathcal{O}$. It follows from $J \mathrm{e}^{\mathrm{i} t h_{0}} J=\mathrm{e}^{-\mathrm{i} t h_{0}}$ and $J \mathrm{e}^{\mathrm{i} t h} J=\mathrm{e}^{-\mathrm{i} t h}$ that $\tau_{0}^{t} \circ j=j \circ \tau_{0}^{-t}$ and $\tau^{t} \circ j=j \circ \tau^{-t}$. A state $\omega$ on $\mathcal{O}$ is time reversal invariant if $\omega \circ j(A)=\omega\left(A^{*}\right)=\overline{\omega(A)}$ for all $A \in \mathcal{O}$. A gauge-invariant quasi-free state with density $\varrho$ such that $J \varrho=\varrho J$ is time reversal invariant

\subsection{NESS of the Electronic Black Box Model}

Let $\omega_{0}$ be a state on $\mathcal{O}$. Following Ruelle ([Ru2]), we say that a state $\omega_{+}$is a nonequilibrium steady state (NESS) of the EBB model associated to $\omega_{0}$ if it is a $w^{*}$-limit point of the net

$$
\left\{\frac{1}{t} \int_{0}^{t} \omega_{0} \circ \tau^{s} \mathrm{~d} s \mid t \geq 0\right\} .
$$

We denote by $\Sigma^{+}\left(\omega_{0}\right)$ the set of NESS associated with $\omega_{0}$. It is easy to show that this set is non-empty and that its elements are $\tau$-invariant states on $\mathcal{O}$. The general structural properties of NESS are described in [JP2], [AJPP].

The following result describes the NESS of the EBB model associated to $\tau_{0}$-invariant gauge-invariant quasi-free states. Its proof will be carried out in Subsection 6.2 using the scattering approach to NESS.

Theorem 3.2 Let $\omega_{0}$ be a gauge-invariant $\tau_{0}^{t}$-invariant quasi-free state on $\mathcal{O}$ with density $\varrho_{0}$. Under Hypotheses (H1)-(H3) the following hold:

1. There is a unique NESS associated to $\omega_{0}$

$$
\Sigma^{+}\left(\omega_{0}\right)=\left\{\omega_{+}\right\}
$$

2. The Møller operators

$$
\Omega_{ \pm}=\mathrm{s}-\lim _{t \rightarrow \pm \infty} \mathrm{e}^{\mathrm{i} t h} \mathrm{e}^{-\mathrm{i} t h_{0}} 1_{\mathrm{ac}}\left(h_{0}\right),
$$


exist and are complete. The restriction $\left.\omega_{+}\right|_{\mathrm{CAR}\left(\mathfrak{h}_{\mathrm{ac}}(h)\right)}$ is the gauge invariant quasifree state with density $\Omega_{-} \varrho_{0} \Omega_{-}^{*}$. For any $\omega_{0}$-normal state $\eta$ and any observable $A \in \mathrm{CAR}\left(\mathfrak{h}_{\mathrm{ac}}(h)\right)$ one has

$$
\lim _{t \rightarrow \infty} \eta\left(\tau^{t}(A)\right)=\omega_{+}(A)
$$

3. For $\varepsilon \in \mathrm{sp}_{\mathrm{pp}}(h)$ denote by $P_{\varepsilon}$ the corresponding spectral projection. For any trace class operator $c$ on $\mathfrak{h}$ one has

$$
\omega_{+}(\mathrm{d} \Gamma(c))=\operatorname{Tr}\left(\varrho_{+} c\right),
$$

where

$$
\varrho_{+}=\Omega_{-} \varrho_{0} \Omega_{-}^{*}+\sum_{\varepsilon \in \mathrm{sp}_{\mathrm{pp}}(h)} P_{\varepsilon} \varrho_{0} P_{\varepsilon}
$$

Remark. If $\operatorname{sp}_{\mathrm{pp}}(h)=\emptyset$ then

$$
\lim _{t \rightarrow \infty} \eta\left(\tau^{t}(A)\right)=\omega_{+}(A)
$$

holds for any $\omega_{0}$-normal state $\eta$ and any $A \in \mathcal{O}$. However, this limit does not exist for all $A \in \mathcal{O}$ if $h$ has some eigenvalues and time averaging is necessary to reach a steady state.

\subsection{Flux Observables}

At the one-particle level, a conserved quantity of the reservoirs is a (possibly unbounded) self-adjoint operator $q$ on $\mathfrak{h}$ such that

$$
\mathrm{e}^{\mathrm{i} t h_{0}} q \mathrm{e}^{-\mathrm{i} t h_{0}}=q
$$

for all $t \in \mathbb{R}$. For an ideal Fermi gas the corresponding extensive thermodynamic quantity is given by the second quantization $Q=\mathrm{d} \Gamma(q)$. Here are few examples:

1. The total energy of the $k$-th reservoir is $H_{k}=\mathrm{d} \Gamma\left(h_{k}\right)$.

2. Denote by $1_{k}$ the canonical projection of $\mathfrak{h}$ onto $\mathfrak{h}_{k}$ corresponding to the decomposition (3.2). The total number of Fermions in the $k$-th reservoir is $N_{k}=\mathrm{d} \Gamma\left(1_{k}\right)$.

3. If each Fermion of the system carries a charge $e$, the total charge of the $k$-th reservoir is $Q_{k}=\mathrm{d} \Gamma\left(e 1_{k}\right)=e N_{k}$.

4. Let $\sigma$ be the spin operator of a single Fermion and assume that $\left[h_{0}, \sigma\right]=0$. The total spin of the $k$-th reservoir is $\boldsymbol{\Sigma}_{k}=\mathrm{d} \Gamma\left(\sigma 1_{k}\right)$. 
Such extensive quantities are not elements of $\mathcal{O}$ because the corresponding $q$ 's are not trace class. Their quasi-free expectations $\omega_{\varrho}(\mathrm{d} \Gamma(q))$ are usually ill-defined or infinite because even $\varrho q$ is not trace-class. This is physically consistent with the fact that a spatially extended reservoir at positive density $(0<\varrho<I)$ contains infinitely many particles. Of special interest are the rates of change of conserved extensive quantities, formally given by

$$
\Phi_{q}=\mathrm{d} \Gamma\left(\varphi_{q}\right), \quad \varphi_{q}=-\left.\frac{\mathrm{d}}{\mathrm{d} t} \mathrm{e}^{\mathrm{i} t h} q \mathrm{e}^{-\mathrm{i} t h}\right|_{t=0}=-\mathrm{i}[h, q] .
$$

$\Phi_{q}$ measures the flux of $Q=\mathrm{d} \Gamma(q)$ entering the system $\mathcal{S}$ from the reservoirs $\mathcal{R}$. Depending on $q$ and on the junctions, the flux observable $\Phi_{q}$ may or may not be an element of $\mathcal{O}$. In the latter case, in order to compute its expectation we need to perform some regularization.

For $\eta>0$ set $f_{\eta}(\varepsilon)=\varepsilon(1+\eta \varepsilon)^{-(p+1)}$ and define the regularized flux by

$$
\Phi_{q}^{\eta}=\mathrm{d} \Gamma\left(\varphi_{q}^{\eta}\right), \quad \varphi_{q}^{\eta}=-\mathrm{i}\left[f_{\eta}(h), q\right] .
$$

By Lemma 3.1, under Hypotheses $(\mathrm{H} 1)-(\mathrm{H} 2), f_{\eta}(h)-f_{\eta}\left(h_{0}\right)$ is trace class for any sufficiently small $\eta>0$. Therefore, if $q$ is bounded then its regularized flux

$$
\varphi_{q}^{\eta}=-\mathrm{i}\left[f_{\eta}(h)-f_{\eta}\left(h_{0}\right), q\right]
$$

is trace class and we define the expectation of $\Phi_{q}$ as

$$
\omega_{\varrho}\left(\Phi_{q}\right)=\lim _{\eta \rightarrow 0} \omega_{\varrho}\left(\Phi_{q}^{\eta}\right)=\lim _{\eta \rightarrow 0} \operatorname{Tr}\left(\varrho \varphi_{q}^{\eta}\right),
$$

whenever this limit exists. Note that for $p=-1$ one has $f_{\eta}(\varepsilon)=\varepsilon$. In this case it is not necessary to regularize the flux $\varphi_{q}$ of bounded $q$.

To be able to handle heat fluxes we have to extend Definition (3.6) to unbounded $q$.

Definition 3.3 A tempered conserved charge is a self-adjoint operator $q$ on $\mathfrak{h}$ satisfying (3.4) and such that $q^{(\Lambda)}=q 1_{[-\infty, \Lambda]}\left(h_{0}\right)$ is bounded for all $\Lambda \in \mathbb{R}$.

We define the expectation of the flux of a tempered conserved charge $q$ by

$$
\omega_{\varrho}\left(\Phi_{q}\right)=\lim _{\Lambda \rightarrow \infty} \omega_{\varrho}\left(\Phi_{q^{(\Lambda)}}\right)=\lim _{\Lambda \rightarrow \infty} \lim _{\eta \rightarrow 0} \omega_{\varrho}\left(\Phi_{q^{(\Lambda)}}^{\eta}\right)=\lim _{\Lambda \rightarrow \infty} \lim _{\eta \rightarrow 0} \operatorname{Tr}\left(\varrho \varphi_{q^{(\Lambda)}}^{\eta}\right)
$$

whenever these limits exist.

On physical grounds we expect fluxes to have zero mean at equilibrium.

Lemma 3.4 If $q$ is a tempered conserved charge and $\omega$ a state with density $\varrho=F(h)$ such that $F(h)-F\left(h_{0}\right)$ belongs to the trace class then one has

$$
\omega\left(\Phi_{q}\right)=0 .
$$

In particular, under Assumptions (H1)-(H2) steady currents vanish at thermal equilibrium. 
Proof. To prove the first assertion, it suffices to show that $\omega\left(\Phi_{q^{(\Lambda)}}^{\eta}\right)=0$ for any $\eta, \Lambda$. Using the cyclicity of the trace, we can write

$$
\begin{aligned}
\omega\left(\Phi_{q^{(\Lambda)}}^{\eta}\right) & =\operatorname{Tr}\left(F(h) \mathrm{i}\left[q^{(\Lambda)}, f_{\eta}(h)-f_{\eta}\left(h_{0}\right)\right]\right) \\
& =\operatorname{Tr}\left(\left(f_{\eta}(h)-f_{\eta}\left(h_{0}\right)\right) \mathrm{i}\left[F(h), q^{(\Lambda)}\right]\right) \\
& =\operatorname{Tr}\left(\left(f_{\eta}(h)-f_{\eta}\left(h_{0}\right)\right) \mathrm{i}\left[F(h)-F\left(h_{0}\right), q^{(\Lambda)}\right]\right) \\
& =\operatorname{Tr}\left(f_{\eta}(h) \mathrm{i}\left[F(h)-F\left(h_{0}\right), q^{(\Lambda)}\right]\right)-\operatorname{Tr}\left(f_{\eta}\left(h_{0}\right) \mathrm{i}\left[F(h)-F\left(h_{0}\right), q^{(\Lambda)}\right]\right) .
\end{aligned}
$$

The second term on the right hand side of the last identity vanishes since

$$
f_{\eta}\left(h_{0}\right) \mathrm{i}\left[F(h)-F\left(h_{0}\right), q^{(\Lambda)}\right]=\mathrm{i}\left[f_{\eta}\left(h_{0}\right)\left(F(h)-F\left(h_{0}\right)\right), q^{(\Lambda)}\right],
$$

and since $F(h)-F\left(h_{0}\right)$ is trace class. Thus, using again the cyclicity of the trace, we obtain

$$
\begin{aligned}
\omega\left(\Phi_{q^{(\Lambda)}}^{\eta}\right) & =\operatorname{Tr}\left(f_{\eta}(h) \mathrm{i}\left[F(h)-F\left(h_{0}\right), q^{(\Lambda)}\right]\right) \\
& =\operatorname{Tr}\left(q^{(\Lambda)} \mathrm{i}\left[f_{\eta}(h), F(h)-F\left(h_{0}\right)\right]\right) \\
& =\operatorname{Tr}\left(q^{(\Lambda)} \mathrm{i}\left[F\left(h_{0}\right), f_{\eta}(h)\right]\right) \\
& =\operatorname{Tr}\left(q^{(\Lambda)} \mathrm{i}\left[F\left(h_{0}\right), f_{\eta}(h)-f_{\eta}\left(h_{0}\right)\right]\right) \\
& =\operatorname{Tr}\left(\mathrm{i}\left[F\left(h_{0}\right), q^{(\Lambda)}\left(f_{\eta}(h)-f_{\eta}\left(h_{0}\right)\right)\right]\right) .
\end{aligned}
$$

The last expression vanishes since $f_{\eta}(h)-f_{\eta}\left(h_{0}\right)$ is trace class.

To prove the second assertion note that if $\omega$ is a thermal equilibrium state then its density is given by $F(h)=\left(1+\mathrm{e}^{\beta(h-\mu)}\right)^{-1}$ for some $\beta>0$ and $\mu \in \mathbb{R}$. It is easily seen that there exists a function $\varphi \in C_{0}^{\infty}(\mathbb{R})$ such that $F(h)=\varphi\left(\left(1+h+E_{0}\right)^{-p}\right)$. Thus, it follows from Assumptions (H1)-(H2) and Lemma 3.1 that $F(h)-F\left(h_{0}\right)$ is trace class

The following lemma shows that, as far as flux observables are concerned, the NESS $\omega_{+}$ describes the asymptotics of a large class of initial states. Moreover the NESS expectations of fluxes are insensitive to the point spectrum of $h$.

Proposition 3.5 Let $q$ be a tempered conserved charge. Under the assumptions of Theorem 3.2 one has

$$
\omega_{+}\left(\Phi_{q^{(\Lambda)}}^{\eta}\right)=\operatorname{Tr}\left(\Omega_{-} \varrho_{0} \Omega_{-}^{*} \varphi_{q^{(\Lambda)}}^{\eta}\right)
$$

and hence

$$
\omega_{+}\left(\Phi_{q}\right)=\lim _{\Lambda \rightarrow \infty} \lim _{\eta \rightarrow 0} \operatorname{Tr}\left(\Omega_{-} \varrho_{0} \Omega_{-}^{*} \varphi_{q^{(\Lambda)}}^{\eta}\right) .
$$

Moreover, if $\omega$ is a state on $\mathcal{O}$ with density $\varrho$ such that $\left(\varrho-\varrho_{0}\right) 1_{\mathrm{ac}}(h)$ is compact $(\omega$ does not need to be quasi-free), then

$$
\lim _{t \rightarrow \infty} \frac{1}{t} \int_{0}^{t} \omega \circ \tau^{s}\left(\Phi_{q^{(\Lambda)}}^{\eta}\right) \mathrm{d} s=\omega_{+}\left(\Phi_{q^{(\Lambda)}}^{\eta}\right)
$$


Proof. By Part 3 of Theorem 3.2 the first statement follows from the obvious fact that

$$
P_{\varepsilon} \varphi_{q^{(\Lambda)}}^{\eta} P_{\varepsilon}=-\mathrm{i} P_{\varepsilon}\left[f_{\eta}(h), q^{(\Lambda)}\right] P_{\varepsilon}=-\mathrm{i} P_{\varepsilon}\left[f_{\eta}(\varepsilon), q^{(\Lambda)}\right] P_{\varepsilon}=0
$$

for any $\varepsilon \in \operatorname{sp}_{\mathrm{pp}}(h)$.

To prove the second statement we decompose

$$
\omega \circ \tau^{t}\left(\Phi_{q^{(\Lambda)}}^{\eta}\right)=\operatorname{Tr}\left(\varrho \mathrm{e}^{\mathrm{i} t h} \varphi_{q^{(\Lambda)}}^{\eta} \mathrm{e}^{-\mathrm{i} t h}\right)=\operatorname{Tr}\left(\varrho_{0} \mathrm{e}^{\mathrm{i} t h} \varphi_{q^{(\Lambda)}}^{\eta} \mathrm{e}^{-\mathrm{i} t h}\right)+\operatorname{Tr}\left(\left(\varrho-\varrho_{0}\right) \mathrm{e}^{\mathrm{i} t h} \varphi_{q^{(\Lambda)}}^{\eta} \mathrm{e}^{-\mathrm{i} t h}\right) .
$$

By Theorem 3.2 the first term on the right hand side of this identity converges, in timemean, to $\omega_{+}\left(\Phi_{q^{(\Lambda)}}^{\eta}\right)$. The second term can be further decomposed, as in the proof of Part 1 of Theorem 3.2, into four terms

$$
\operatorname{Tr}\left(\left(\varrho-\varrho_{0}\right) \mathrm{e}^{\mathrm{i} t h} \varphi_{q^{(\Lambda)}}^{\eta} \mathrm{e}^{-\mathrm{i} t h}\right)=\sum_{\alpha, \alpha^{\prime} \in\{\mathrm{ac}, \mathrm{pp}\}} \operatorname{Tr}\left(\left(\varrho-\varrho_{0}\right) \mathrm{e}^{\mathrm{i} t h} 1_{\alpha}(h) \varphi_{q^{(\Lambda)}}^{\eta} 1_{\alpha^{\prime}}(h) \mathrm{e}^{-\mathrm{i} t h}\right) .
$$

Due to the compactness of $\left(\varrho-\varrho_{0}\right) 1_{\mathrm{ac}}(h)$ the 3 parts containing an ac-component vanish as $t \rightarrow \infty$. The remaining part converges in time mean to

$$
\sum_{\varepsilon \in \mathrm{sp}_{\mathrm{pp}}(h)} \operatorname{Tr}\left(\left(\varrho-\varrho_{0}\right) P_{\varepsilon} \varphi_{q^{(\Lambda)}}^{\eta} P_{\varepsilon}\right)
$$

which is zero by Equ. (3.8)

\section{Landauer-Büttiker Formulas}

A formula expressing the steady electric current flowing through a one-dimensional sample $\mathcal{S}$ coupled to two reservoirs $\mathcal{R}_{1}$ and $\mathcal{R}_{2}$ was first proposed by Landauer [L]. The characteristic feature of this formula is that it only refers to the internal structure of $\mathcal{S}$ through the scattering data (transmission probability) of the corresponding one-electron problem. Analogous formulas were later obtained for other geometries (e.g., two multichannel reservoirs [FL], [LA], [BILP] or more than two reservoirs [B1], [B2]) and for heat currents ([AE], $[\mathrm{SI}])$. The resulting scattering approach to electronic transport is nowadays coined as "Landauer-Büttiker formalism" (see [D],[IL] for reviews and more complete references to the enormous bibliography on the subject).

\subsection{Steady Currents}

In this subsection we formulate our main result - Theorem 4.1 - which substantiates the relation between the above mentioned Landauer-Büttiker formalism and Ruelle's scattering approach to NESS. In the latter framework, steady state currents are just expectations of flux observables in the NESS, and so the starting point of the proof 
of Theorem 4.1 is Formula (3.7) of Proposition 3.5. Due to the general nature of the Electronic Black Box Model this proof is quite involved and postponed to Subsection 6.3.

To formulate our result, let us introduce some notation. There exists a measurable family of Hilbert spaces $\left\{\mathfrak{h}(\varepsilon) \mid \varepsilon \in \operatorname{sp}_{\mathrm{ac}}\left(h_{0}\right)\right\}$ and a unitary operator

$$
U: \mathfrak{h}_{\mathrm{ac}}\left(h_{0}\right) \rightarrow \int_{\mathrm{sp}_{\mathrm{ac}}\left(h_{0}\right)}^{\oplus} \mathfrak{h}(\varepsilon) \mathrm{d} \varepsilon,
$$

such that $U h_{0} U^{*}$ is the operator of multiplication by $\varepsilon$ on each fiber $\mathfrak{h}(\varepsilon)$. Moreover, if a bounded operator $a \in \mathcal{B}(\mathfrak{h})$ commutes with $h_{0}$ then there exists a measurable family $a(\varepsilon) \in \mathcal{B}(\mathfrak{h}(\varepsilon))$ such that $U a U^{*}$ acts by multiplication with $a(\varepsilon)$ on each fiber $\mathfrak{h}(\varepsilon)$. This applies to the initial density operator $\varrho_{0}$ as well as to the scattering matrix $S=\Omega_{+}^{*} \Omega_{-}$. Similarly, if $q$ is a tempered conserved charge then $U q U^{*}$ acts by multiplication by a bounded operator $q(\varepsilon)$ on $\mathfrak{h}(\varepsilon)$. In physical terms, the Hilbert space $\mathfrak{h}(\varepsilon)$ is the energyshell. Its dimension is the number of open channels at energy $\varepsilon$ and $S(\varepsilon)$ is the on-shell scattering matrix.

Theorem 4.1 Under Assumptions (H1)-(H3), if $q$ is a tempered conserved charge such that

$$
\underset{\varepsilon \in \operatorname{sp}_{\mathrm{ac}}\left(h_{0}\right)}{\operatorname{ess}-\sup }\left(1+\varepsilon+E_{0}\right)^{p+1}\left\|\varrho_{0}(\varepsilon)\right\|\|q(\varepsilon)\|<\infty
$$

then

$$
\begin{aligned}
\omega_{+}\left(\Phi_{q}\right) & =\lim _{\Lambda \rightarrow \infty} \lim _{\eta \rightarrow 0} \omega_{+}\left(\Phi_{q^{(\Lambda)}}^{\eta}\right) \\
& =\int_{\operatorname{sp}_{\mathfrak{a c}}\left(h_{0}\right)} \operatorname{Tr}_{\mathfrak{h}(\varepsilon)}\left(\varrho_{0}(\varepsilon)\left[q(\varepsilon)-S(\varepsilon)^{*} q(\varepsilon) S(\varepsilon)\right]\right) \frac{\mathrm{d} \varepsilon}{2 \pi} .
\end{aligned}
$$

The integral in (4.11) is absolutely convergent.

Due to our rather general setup, Formula (4.11) cannot be immediately recognized as the usual Landauer-Büttiker formula for steady currents. To rewrite it in a more familiar form we assume:

(H4) The sample system Hamiltonian $h_{\mathcal{S}}$ has purely discrete spectrum.

Under Hypothesis (H4) it immediately follows from Proposition 3.5 that expectations of flux observables in the NESS $\omega_{+}$are independent of the initial state of the system $\mathcal{S}$. According to the decompositions (3.2) and (3.3), one has now

$$
\mathfrak{h}_{\mathrm{ac}}\left(h_{0}\right)=\bigoplus_{k=1}^{M} \mathfrak{h}_{\mathrm{ac}}\left(h_{k}\right), \quad \mathfrak{h}(\varepsilon)=\bigoplus_{k=1}^{M} \mathfrak{h}_{k}(\varepsilon),
$$


and the on-shell scattering matrix $S(\varepsilon)$ has a corresponding $M \times M$-matrix structure

$$
S_{j k}(\varepsilon)=\delta_{j k}+t_{j k}(\varepsilon) .
$$

For $j \neq k$ the operator $t_{j k}(\varepsilon): \mathfrak{h}_{k}(\varepsilon) \rightarrow \mathfrak{h}_{j}(\varepsilon)$ is the "transmission amplitude" from $\mathcal{R}_{k}$ to $\mathcal{R}_{j}$. The corresponding "transmission probability" is $t_{j k}(\varepsilon)^{*} t_{j k}(\varepsilon)$. In the physics literature, eigenvectors of this operator are called "eigenchannels". The "total transmission probability" is given by

$$
T_{j k}(\varepsilon)=\operatorname{Tr}_{\mathfrak{h}_{k}(\varepsilon)}\left(t_{j k}(\varepsilon)^{*} t_{j k}(\varepsilon)\right) .
$$

Note that $T_{j k}(\varepsilon) \geq 0$ and that $T_{j k}(\varepsilon)=0$ for $\varepsilon \notin \operatorname{sp}_{\mathrm{ac}}\left(h_{k}\right) \cap \mathrm{sp}_{\mathrm{ac}}\left(h_{j}\right)$.

Corollary 4.2 Assume that Hypotheses (H1)-(H4) hold and that the initial state of the reservoirs $\mathcal{R}$ is given by

$$
\varrho_{0, \mathcal{R}}=\bigoplus_{k=1}^{M} f_{k}\left(h_{k}\right)
$$

Let

$$
q=\bigoplus_{j=1}^{M} g_{j}\left(h_{j}\right)
$$

and assume that

$$
\max _{k j}\left(\operatorname{ess}_{\varepsilon \in \operatorname{sp}_{\mathrm{ac}}\left(h_{k}\right) \cap \sup _{\mathrm{ac}}\left(h_{j}\right)}\left(1+\varepsilon+E_{0}\right)^{p+1}\left|f_{k}(\varepsilon)\right|\left|g_{j}(\varepsilon)\right|\right)<\infty .
$$

Then, one has

$$
\begin{aligned}
\omega_{+}\left(\Phi_{q}\right) & =\sum_{k, j=1}^{M} \int_{\operatorname{sp}_{\mathrm{ac}}\left(h_{k}\right) \cap \mathrm{sp}_{\mathrm{ac}}\left(h_{j}\right)} T_{j k}(\varepsilon)\left(f_{j}(\varepsilon)-f_{k}(\varepsilon)\right) g_{j}(\varepsilon) \frac{\mathrm{d} \varepsilon}{2 \pi}, \\
& =\sum_{k, j=1}^{M} \int_{\operatorname{sp}_{\mathrm{ac}}\left(h_{k}\right) \cap \mathrm{sp}_{\mathrm{ac}}\left(h_{j}\right)} T_{j k}(\varepsilon) f_{k}(\varepsilon)\left(g_{k}(\varepsilon)-g_{j}(\varepsilon)\right) \frac{\mathrm{d} \varepsilon}{2 \pi},
\end{aligned}
$$

where the integrands are absolutely integrable.

Proof. Inserting Definition (4.12) into Formula (4.11) leads to

$$
\begin{array}{r}
\omega_{+}\left(\Phi_{q}\right)=-\sum_{k=1}^{M} \int_{\mathrm{sp}_{\mathrm{ac}}\left(h_{k}\right)} f_{k}(\varepsilon) \operatorname{Tr}_{\mathfrak{h}_{k}(\varepsilon)}\left(\left(t_{k k}(\varepsilon)+t_{k k}(\varepsilon)^{*}\right) g_{k}(\varepsilon)\right. \\
\left.+\sum_{j=1}^{M} t_{j k}(\varepsilon)^{*} g_{j}(\varepsilon) t_{j k}(\varepsilon)\right) \frac{\mathrm{d} \varepsilon}{2 \pi} .
\end{array}
$$


The unitarity of $S(\varepsilon)$ yields the optical theorem

$$
\sum_{l=1}^{M} t_{j l}(\varepsilon) t_{k l}(\varepsilon)^{*}=\sum_{l=1}^{M} t_{l j}(\varepsilon)^{*} t_{l k}(\varepsilon)=-\left(t_{j k}(\varepsilon)+t_{k j}(\varepsilon)^{*}\right) .
$$

Using this relation, Equ. (4.15) is easily recast into the form (4.14). To obtain the form (4.13) it suffices to notice that, due to the first equality in (4.16) and to the cyclicity of the trace one has

$$
\sum_{j=1}^{M} T_{j k}(\varepsilon)=\sum_{j=1}^{M} T_{k j}(\varepsilon)
$$

The absolute integrability of the integrands in (4.13) and (4.14) follows from the estimate (6.49) below.

Remark. Corollary 4.2 has two interesting immediate consequences. First, if all the reservoirs are in the "same state", i.e., if $f_{1}=\cdots=f_{M}$, then the steady currents vanish. Second, if $g_{1}=\cdots=g_{M}=g$ then the corresponding $\Phi_{q}$ is the total $g\left(h_{0}\right)$-flux entering the sample $\mathcal{S}$ and

$$
\omega_{+}\left(\Phi_{q}\right)=\sum_{k=1}^{M} \omega_{+}\left(\Phi_{g\left(h_{k}\right)}\right)=0,
$$

is the corresponding Kirchoff rule.

\subsection{Entropy Production}

From now on we restrict ourselves to thermal initial states, that is, states of the form

$$
T_{0, \mathcal{R}}=\bigoplus_{k=1}^{M} f_{\beta_{k} \mu_{k}}\left(h_{k}\right), \quad f_{\beta \mu}(\varepsilon)=\left(1+\mathrm{e}^{\beta(\varepsilon-\mu)}\right)^{-1},
$$

which describe the physically important situation where each reservoir $\mathcal{R}_{k}$ is at thermal equilibrium at inverse temperature $\beta_{k}>0$ and chemical potential $\mu_{k} \in \mathbb{R}$. We further denote by

$$
\Phi_{k}^{\mathrm{h}}=\mathrm{d} \Gamma\left(\varphi_{k}^{\mathrm{h}}\right), \quad \varphi_{k}^{\mathrm{h}}=-\mathrm{i}\left[h, h_{k}\right],
$$

the heat current out of $\mathcal{R}_{k}$ and by

$$
\Phi_{k}^{\mathrm{c}}=\mathrm{d} \Gamma\left(\varphi_{k}^{\mathrm{c}}\right), \quad \varphi_{k}^{\mathrm{c}}=-\mathrm{i}\left[h, 1_{k}\right],
$$

the charge current (corresponding respectively to Example 1 and 3 in Subsection 3.2, where we have set the charge of a single Fermion to 1). In terms of these currents, the entropy production rate observable is given by

$$
\sigma=-\sum_{k=1}^{M} \beta_{k}\left(\Phi_{k}^{\mathrm{h}}-\mu_{k} \Phi_{k}^{\mathrm{c}}\right)
$$


(see e.g. $[\mathrm{AJPP}]$ ) and the mean entropy production rate in the NESS $\omega_{+}$is

$$
\operatorname{Ep}\left(\omega_{+}\right)=\omega_{+}(\sigma) .
$$

Remark 1. In the case of local perturbations (the case $p=-1$ in Hypothesis (H2)) it follows from the entropy balance identity (see [JP2]) that, with the above definition, one has

$$
\operatorname{Ep}\left(\omega_{+}\right)=-\lim _{t \rightarrow \infty} \frac{1}{t} \operatorname{Ent}\left(\omega_{\varrho_{0}} \circ \tau^{t} \mid \omega_{\varrho_{0}}\right),
$$

where $\operatorname{Ent}(\cdot \mid \cdot)$ denotes the Araki relative entropy (with the notational convention of $[\mathrm{BR}])$. In this case the inequality $\operatorname{Ep}\left(\omega_{+}\right) \geq 0$ is a direct consequence of the fact that the Araki relative entropy is non-positive. At the current level of generality such an interpretation is not possible for $p>0$ since the states $\omega_{\varrho_{0}} \circ \tau^{t}$ are not necessarily $\omega_{\varrho_{0}}{ }^{-}$ normal for $t \neq 0$. We shall see however (Proposition 4.4) that $\operatorname{Ep}\left(\omega_{+}\right) \geq 0$ holds in this case too.

Remark 2. From a physical point of view the initial states $\omega_{\varrho_{0}}$ specified by Equ. (4.18) are not very natural in the case $p>0$. On the other hand, in typical physically relevant models where $p>0$ local compactness holds in the following sense. The one-particle Hilbert space is given by $\mathfrak{h}=L^{2}(D, \mathrm{~d} x)$ for some unbounded domain $D \subset \mathbb{R}^{d}$. For any compact region $O \subset D$ the operator of multiplication by characteristic function $1_{O}(x)$ is $h_{0}$-compact and $h$-compact. Under such circumstances it follows from the last assertion of Proposition 3.5 that any initial state with density $\varrho$ such that

$$
\left(\varrho-\varrho_{0, \mathcal{R}}\right)\left(I-1_{O}(x)\right) \text { and } \varrho 1_{O}(x) \text {, }
$$

are compact for some compact $O \subset D$ containing $\mathcal{S}$ induces the same steady currents as $\omega_{\varrho_{0}}$.

Remark 3. A change of Hamiltonians

$$
h_{0} \mapsto h_{0}^{\prime}=h_{0}+E, \quad h \mapsto h^{\prime}=h+E,
$$

combined with the corresponding change of thermodynamic parameters

$$
\beta_{k} \mapsto \beta_{k}^{\prime}=\beta_{k}, \quad \mu_{k} \mapsto \mu_{k}^{\prime}=\mu_{k}+E,
$$

for some $E \in \mathbb{R}$ does not affect the NESS $\omega_{+}$. It does however affect the flux observables which change according to

$$
\begin{aligned}
& \varphi_{k}^{\mathrm{h}} \mapsto \varphi_{k}^{\mathrm{h}}=-\mathrm{i}\left[h+E, h_{k}+E 1_{k}\right]=\varphi_{k}^{\mathrm{h}}+E \varphi_{k}^{\mathrm{c}}, \\
& \varphi_{k}^{\mathrm{c}} \mapsto \varphi^{\prime \mathrm{c}}{ }_{k}=-\mathrm{i}\left[h+E, 1_{k}\right]=\varphi_{k}^{\mathrm{c}} .
\end{aligned}
$$


Entropy production rate remains unchanged since

$$
\sigma^{\prime}=-\sum_{k=1}^{M} \beta_{k}^{\prime}\left(\Phi_{k}^{\prime \mathrm{h}}-\mu_{k}^{\prime} \Phi_{k}^{\prime \mathrm{c}}\right)=-\sum_{k=1}^{M} \beta_{k}\left(\Phi_{k}^{\mathrm{h}}-\mu_{k} \Phi_{k}^{\mathrm{c}}\right)=\sigma .
$$

Applying Corollary 4.2 we immediately obtain the following.

Corollary 4.3 Under Hypotheses (H1)-(H4), if $\varrho_{0, \mathcal{R}}$ has the form (4.18) then one has

$$
\begin{aligned}
\omega_{+}\left(\Phi_{k}^{\mathrm{c}}\right) & =\sum_{j=1}^{M} \int_{\mathrm{sp}_{\mathrm{ac}}\left(h_{k}\right) \cap \mathrm{sp}_{\mathrm{ac}}\left(h_{j}\right)} T_{k j}(\varepsilon)\left(f_{\beta_{k} \mu_{k}}(\varepsilon)-f_{\beta_{j} \mu_{j}}(\varepsilon)\right) \frac{\mathrm{d} \varepsilon}{2 \pi} \\
\omega_{+}\left(\Phi_{k}^{\mathrm{h}}\right) & =\sum_{j=1}^{M} \int_{\mathrm{sp}_{\mathrm{ac}}\left(h_{k}\right) \cap \mathrm{sp}_{\mathrm{ac}}\left(h_{j}\right)} \varepsilon T_{k j}(\varepsilon)\left(f_{\beta_{k} \mu_{k}}(\varepsilon)-f_{\beta_{j} \mu_{j}}(\varepsilon)\right) \frac{\mathrm{d} \varepsilon}{2 \pi} \\
\operatorname{Ep}\left(\omega_{+}\right) & =\sum_{k, j=1}^{M} \int_{\mathrm{sp}_{\mathrm{ac}}\left(h_{k}\right) \cap \mathrm{sp}_{\mathrm{ac}}\left(h_{j}\right)} \xi_{k}(\varepsilon) T_{k j}(\varepsilon)\left(F\left(\xi_{j}(\varepsilon)\right)-F\left(\xi_{k}(\varepsilon)\right)\right) \frac{\mathrm{d} \varepsilon}{2 \pi}
\end{aligned}
$$

where $\xi_{k}(\varepsilon)=\beta_{k}\left(\varepsilon-\mu_{k}\right)$ and $F(x)=\left(1+\mathrm{e}^{x}\right)^{-1}$.

By the remark after Corollary 4.2 one has the Kirchoff rules

$$
\sum_{k=1}^{M} \omega_{+}\left(\Phi_{k}^{\mathrm{h}}\right)=0, \quad \sum_{k=1}^{M} \omega_{+}\left(\Phi_{k}^{\mathrm{c}}\right)=0
$$

which express respectively the first law of thermodynamics (energy conservation) and charge conservation. The next result expresses the second law of thermodynamics.

Proposition 4.4 Under the assumptions of Corollary 4.3 one has

$$
\operatorname{Ep}\left(\omega_{+}\right) \geq \frac{1}{M} \int_{\mathrm{sp}_{\mathrm{ac}}\left(h_{k}\right) \cap \mathrm{sp}_{\mathrm{ac}}\left(h_{j}\right)} T_{k j}(\varepsilon) F\left(\left|\xi_{k}(\varepsilon)\right|\right) F\left(\left|\xi_{j}(\varepsilon)\right|\right)\left(\xi_{k}(\varepsilon)-\xi_{j}(\varepsilon)\right)^{2} \frac{\mathrm{d} \varepsilon}{2 \pi} \geq 0
$$

for any pair $(k, j)$.

We say that the channel $j \rightarrow k$ is open if the set

$$
\left\{\varepsilon \in \operatorname{sp}_{\mathrm{ac}}\left(h_{k}\right) \cap \operatorname{sp}_{\mathrm{ac}}\left(h_{j}\right) \mid T_{k j}(\varepsilon) \neq 0\right\},
$$

has positive Lebesgue measure. The following result is an immediate consequence of Proposition 4.4 . 
Theorem 4.5 If there exists an open channel $j \rightarrow k$ such that either $\beta_{j} \neq \beta_{k}$ or $\mu_{j} \neq \mu_{k}$ then $\operatorname{Ep}\left(\omega_{+}\right)>0$.

We shall prove Proposition 4.4 in Subsection 6.4 using the unitarity of the $S$-matrix. As far as we know and according to Stückelberg [S] the idea of deriving positivity of entropy production (or Boltzmann's $H$-Theorem) from the unitarity of the scattering matrix goes back to Pauli. Our proof follows the implementation of this idea given by Inagaki, Wanders and Piron in [IWP].

If in addition to the hypotheses of Proposition 4.4 Assumption (TRI) also holds, then the proof of positivity of entropy production is considerably simpler [AJPP]. In this case the relation $J \Omega_{ \pm}=\Omega_{\mp} J$ yields $S^{*}=J S J$ and so the transmission probabilities $T_{k j}$ are symmetric, $T_{k j}(\varepsilon)=T_{j k}(\varepsilon)$. Symmetrizing the formula (4.22) we derive the expression

$$
\operatorname{Ep}\left(\omega_{+}\right)=\sum_{k, j=1}^{M} \int_{\mathrm{sp}_{\mathrm{ac}}\left(h_{k}\right) \cap \mathrm{sp}_{\mathrm{ac}}\left(h_{j}\right)} T_{k j}(\varepsilon)\left(\xi_{k}(\varepsilon)-\xi_{j}(\varepsilon)\right)\left(F\left(\xi_{j}(\varepsilon)\right)-F\left(\xi_{k}(\varepsilon)\right)\right) \frac{\mathrm{d} \varepsilon}{4 \pi},
$$

which is obviously non-negative since the function $F$ is decreasing, and is strictly positive under the assumptions of Theorem 4.5. Using the elementary inequality

$$
(F(y)-F(x))(x-y) \geq F(|x|) F(|y|)(x-y)^{2},
$$

we also obtain the lower bound

$$
\operatorname{Ep}\left(\omega_{+}\right) \geq \sum_{k, j} \int_{\operatorname{sp}_{\mathrm{ac}}\left(h_{k}\right) \cap \mathrm{sp}_{\mathrm{ac}}\left(h_{j}\right)} T_{k j}(\varepsilon) F\left(\left|\xi_{k}(\varepsilon)\right|\right) F\left(\left|\xi_{j}(\varepsilon)\right|\right)\left(\xi_{k}(\varepsilon)-\xi_{j}(\varepsilon)\right)^{2} \frac{\mathrm{d} \varepsilon}{4 \pi} .
$$

\subsection{Linear Response}

In nonequilibrium statistical mechanics first order perturbation theory w.r.t. the forces that drive the system out of equilibrium is called linear response theory. In the EBBmodel the driving forces are temperature and chemical potential differentials appearing in the initial density of the reservoir (4.18). Fixing reference inverse temperature $\beta$ and chemical potential $\mu$ we set

$$
\beta_{k}=\beta-X_{k}^{\mathrm{h}}, \quad \beta_{k} \mu_{k}=\beta \mu+X_{k}^{\mathrm{c}},
$$

in Equ. (4.18). We denote $\omega_{X+}$, with $X=\left(X_{1}^{\mathrm{h}}, \ldots, X_{M}^{\mathrm{h}}, X_{1}^{\mathrm{c}}, \ldots, X_{M}^{\mathrm{c}}\right)$, the corresponding NESS. The equilibrium state of the system at inverse temperature $\beta$ and chemical potential $\mu$ is the gauge-invariant quasi-free state $\omega_{\text {eq }}$ with density $f_{\text {eq }}(h)$ where

$$
f_{\mathrm{eq}}(\varepsilon)=\frac{1}{1+\mathrm{e}^{\beta(\varepsilon-\mu)}} .
$$


To first order in the thermodynamic forces $X$, the steady state currents are given by

$$
\omega_{X+}\left(\Phi_{k}^{\mathrm{u}}\right)=\sum_{\mathrm{v} \in\{\mathrm{h}, \mathrm{c}\}} \sum_{j=1}^{M} L_{k j}^{\mathrm{uv}} X_{j}^{\mathrm{v}}+\mathrm{O}\left(|X|^{2}\right),
$$

where the $2 M \times 2 M$-matrix of kinetic transport coefficients $L=\left(L_{k j}^{\mathrm{uv}}\right)$ is given by

$$
L_{k j}^{\mathrm{uv}}=\left.\partial_{X_{j}^{\mathrm{v}}} \omega_{X+}\left(\Phi_{k}^{\mathrm{u}}\right)\right|_{X=0} .
$$

Using the conservation laws (4.23) entropy production can be expressed as

$$
0 \leq \operatorname{Ep}\left(\omega_{X+}\right)=\sum_{\mathrm{u} \in\{\mathrm{h}, \mathrm{c}\}} \sum_{k=1}^{M} \omega_{X+}\left(\Phi_{k}^{\mathrm{u}}\right) X_{k}^{\mathrm{u}}=\langle X, L X\rangle+\mathrm{O}\left(|X|^{2}\right),
$$

which shows that the symmetric part of the matrix $L$ is positive semi-definite on $\mathbb{R}^{M}$.

Proposition 4.6 Under Assumptions (H1)-(H4) the kinetic transport coefficients of the EBB model are given by

$$
L_{k j}^{\mathrm{uv}}=-\int_{\mathrm{sp}_{\mathrm{ac}}\left(h_{0}\right)} \varepsilon^{n_{\mathrm{u}}+n_{\mathrm{v}}} f_{\mathrm{eq}}(\varepsilon)\left(1-f_{\mathrm{eq}}(\varepsilon)\right) D_{k j}(\varepsilon) \frac{\mathrm{d} \varepsilon}{2 \pi},
$$

where $n_{\mathrm{c}}=0, n_{\mathrm{h}}=1$ and

$$
D_{k j}(\varepsilon)=T_{k j}(\varepsilon)-\delta_{k j} \sum_{l=1}^{M} T_{k l}(\varepsilon)
$$

Proof. Since

$$
\partial_{X_{j}^{\mathrm{v}}} f_{\beta_{k} \mu_{k}}(\varepsilon)=\delta_{k j} \varepsilon^{n_{\mathrm{v}}} f_{\beta_{k} \mu_{k}}(\varepsilon)\left(1-f_{\beta_{k} \mu_{k}}(\varepsilon)\right),
$$

the Landauer-Büttiker formulas (4.20), (4.21) and the technical estimate (6.49) below yield

$$
\partial_{X_{j}^{\mathrm{v}}} \omega_{X+}\left(\Phi_{k}^{\mathrm{u}}\right)=-\int_{\mathrm{sp}_{\mathrm{ac}}\left(h_{0}\right)} \varepsilon^{n_{\mathrm{u}}+n_{\mathrm{v}}} f_{\beta_{j} \mu_{j}}(\varepsilon)\left(1-f_{\beta_{j} \mu_{j}}(\varepsilon)\right) D_{k j}(\varepsilon) \frac{\mathrm{d} \varepsilon}{2 \pi},
$$

from which the result follows.

Remark. In the zero-temperature limit $(\beta \rightarrow+\infty)$ Formula (4.25) becomes simply

$$
L_{k j}^{\mathrm{uv}}=-\frac{1}{2 \pi} \mu^{n_{\mathrm{u}}+n_{\mathrm{v}}} D_{k j}(\mu) .
$$


We note that $D_{k k}(\varepsilon) \leq 0$ while $D_{k j}(\varepsilon) \geq 0$ for $k \neq j$. It follows that $L_{k k}^{\text {uu }} \geq 0$ and $L_{k j}^{\mathrm{uu}} \leq 0$ for $k \neq j$. This behavior is not restricted to the linear regime. In fact it follows from Equ. (4.27) that an increase of the thermodynamic force $X_{k}^{\mathrm{u}}$ results in an increase of the conjugated steady current $\omega_{X+}\left(\Phi_{k}^{\mathrm{u}}\right)$ and a decrease of $\omega_{X+}\left(\Phi_{j}^{\mathrm{u}}\right)$ for $j \neq k$.

Another immediate consequence of Proposition 4.6 is the symmetry

$$
L_{k j}^{\mathrm{hc}}=L_{k j}^{\mathrm{ch}}
$$

which holds for any pair $k, j$. A deeper symmetry appears when the system is time reversal invariant.

Proposition 4.7 Under Assumptions (H1)-(H4) and (TRI) the kinetic transport coefficients of the EBB model satisfy the Onsager reciprocity relations

$$
L_{k j}^{\mathrm{hc}}=L_{j k}^{\mathrm{ch}}
$$

Proof. As already noticed in the remark after Proposition 4.4, Assumption (TRI) implies that $T_{j k}(\varepsilon)=T_{k j}(\varepsilon)$ and hence $D_{j k}(\varepsilon)=D_{k j}(\varepsilon)$.

Remark. The transport coefficients $L_{k j}^{\text {uv }}$ are properties of the joint system $\mathcal{S}+\mathcal{R}$. In particular they contain contributions from the junctions connecting the system $\mathcal{S}$ to the reservoirs. To determine the transport coefficients of the sample $\mathcal{S}$ per se is a much more delicate problem, even at the conceptual level. It requires the knowledge of the intensive thermodynamic parameters $\widetilde{\beta}_{k}, \widetilde{\mu}_{k}$ actually applied to $\mathcal{S}$ in the steady state $\omega_{X+}$. Since $\omega_{X+}$ is not an equilibrium state, these local equilibrium parameters are usually not well defined and several approaches have been proposed. One possibility is to compare some spatial averages of the density and energy density, in the NESS $\omega_{X+}$ near the connections to $\mathcal{S}$ with the same averages as in thermal equilibrium. We shall not enter into such details here (see e.g., [AE] and [SI]). Let us simply note that by introducing the local parameters $\widetilde{X}_{k}^{\mathrm{u}}(X)$ defined by

$$
\widetilde{\beta}_{k}=\beta-\widetilde{X}_{k}^{\mathrm{h}}, \quad \widetilde{\beta}_{k} \widetilde{\mu}_{k}=\beta \mu+\widetilde{X}_{k}^{\mathrm{c}},
$$

the transport coefficients of $\mathcal{S}$ can be written as

$$
\widetilde{L}_{k j}^{\mathrm{uv}}=\left.\partial_{\widetilde{X}_{j}^{\mathrm{v}}} \omega_{X+}\left(\Phi_{k}^{\mathrm{u}}\right)\right|_{X=0}=\left.\sum_{l=1}^{M} \sum_{w \in\{\mathrm{h}, \mathrm{c}\}} L_{k l}^{\mathrm{uw}} \frac{\partial X_{l}^{\mathrm{w}}}{\partial \widetilde{X}_{j}^{\mathrm{v}}}\right|_{X=0} .
$$

In some special cases it is possible to express the Jacobian matrix $\partial X_{l}^{\mathrm{w}} /\left.\partial \widetilde{X}_{j}^{\mathrm{v}}\right|_{X=0}$ in terms of scattering data (see [SI] for an example). A noteworthy consequence of such calculations is that the coefficients $\widetilde{L}_{k j}^{\text {uv }}$ do not, in general, satisfy the Onsager relations (4.28). The reader should be warned that, in the physics literature, due to some confusions in the early development of the subject (see e.g., [T] and [IL]), the name "Landauer-Büttiker formula" is given indistinctly to Formula (4.25) for $L_{k j}^{\mathrm{uv}}$ and to the formula obtained from Equ. (4.29). 


\section{Green-Kubo Formulas}

Linear response formulas of the Landauer-Büttiker type discussed in the previous subsection are special features of free Fermi gasses. In the case of interacting Fermions it is generally not possible to express transport coefficients in terms of scattering data (see [MW] for a discussion of this point).

Fluctuation-Dissipation relations express the transport coefficients in terms of integrated current-current correlation functions. They are deeply rooted in the folklore of nonequilibrium statistical mechanics under the generic name "Green-Kubo formulas".

In this subsection we formulate Green-Kubo relations for the locally coupled EBBmodel, i.e., in the case where $v=h-h_{0}$ is trace class $(p=-1$ in Hypothesis (H2)). The proof is deferred to Subsection 6.5.

In order for current-current correlation functions $t \mapsto \omega_{\text {eq }}\left(\tau^{t}\left(\Phi_{k}^{\mathrm{u}}\right) \Phi_{j}^{\mathrm{v}}\right)$ to be well defined we shall assume

(H5) Ran $v \subset \operatorname{Dom} h_{0}$;

which ensures that the fluxes

$$
\begin{aligned}
& \varphi_{k}^{\mathrm{h}}=-\mathrm{i}\left[h, h_{k}\right]=\mathrm{i}\left[h_{k}, v\right]=\mathrm{i}\left(1_{k} h_{0} v-v h_{0} 1_{k}\right), \\
& \varphi_{k}^{\mathrm{c}}=-\mathrm{i}\left[h, 1_{k}\right]=\mathrm{i}\left[1_{k}, v\right],
\end{aligned}
$$

are trace class.

The equilibrium state $\omega_{\text {eq }}$ is a $(\tau, \beta)$-KMS state on the $C^{*}$-algebra $\mathrm{CAR}_{\vartheta}(\mathfrak{h})$. Therefore the correlation functions $t \mapsto \omega_{\text {eq }}\left(\Phi_{k}^{\mathrm{u}} \tau^{t}\left(\Phi_{j}^{\mathrm{v}}\right)\right)$ extend to analytic functions on the strip $S_{\beta}=\{z \in \mathbb{C} \mid 0<\operatorname{Im} z<\beta\}$ which are continuous on the closure $\bar{S}_{\beta}$. We denote these extensions by $z \mapsto \omega_{\text {eq }}\left(\Phi_{k}^{\mathrm{u}} \tau^{z}\left(\Phi_{j}^{\mathrm{v}}\right)\right)$.

Proposition 5.1 Under Assumptions (H1)-(H5) with $p=-1$ one has

$$
L_{k j}^{\mathrm{uv}}=\lim _{t \rightarrow \infty} \frac{1}{\beta} \int_{0}^{t} \mathrm{~d} s \int_{0}^{\beta} \mathrm{d} \theta \omega_{\mathrm{eq}}\left(\tau^{s}\left(\Phi_{k}^{\mathrm{u}}\right) \tau^{\mathrm{i} \theta}\left(\Phi_{j}^{\mathrm{v}}\right)\right) .
$$

If in addition Assumption (TRI) holds then

$$
L_{k j}^{\mathrm{uv}}=\lim _{t \rightarrow \infty} \frac{1}{2} \int_{-t}^{t} \mathrm{~d} s \omega_{\mathrm{eq}}\left(\tau^{s}\left(\Phi_{k}^{\mathrm{u}}\right) \Phi_{j}^{\mathrm{v}}\right)
$$

Remark. The Green-Kubo relations (5.30), (5.31) have been recently derived for more general classes of open quantum systems in [J0P1]-[J0P4]. The proof we give in this paper differs from the general argument of [J0P1]-[J0P4] and uses explicitly the specific structure of the EBB model. 


\section{Proofs}

For notational simplicity (and without loss of generality) we shall assume that $E_{0}=0$ in Assumptions (H1)-(H2).

\subsection{Proof of Lemma 3.1}

Step 1. We first claim that, under Assumptions (H1)-(H2), $(1+h)^{-q}-\left(1+h_{0}\right)^{-q}$ is trace class for any $q \in \mathbb{R}$ such that $q \geq p$. Set $A_{1}=(1+h)^{-p}, A_{0}=\left(1+h_{0}\right)^{-p}$ and note that $0<A_{k} \leq I$.

For $0<s<1$ the formula

$$
x^{s}=\frac{\sin \pi s}{\pi} \int_{0}^{\infty} \frac{x}{x+t} t^{s-1} \mathrm{~d} t
$$

holds for $x>0$ and yields

$$
A_{1}^{1+s}-A_{0}^{1+s}=\frac{\sin \pi s}{\pi} \int_{0}^{\infty}\left(\frac{A_{1}}{A_{1}+t}\left(A_{1}-A_{0}\right) \frac{A_{0} / 2+t}{A_{0}+t}+\frac{A_{1} / 2+t}{A_{1}+t}\left(A_{1}-A_{0}\right) \frac{A_{0}}{A_{0}+t}\right) t^{s-1} \mathrm{~d} t .
$$

The inequalities

$$
\left\|\frac{A_{k}}{A_{k}+t}\right\| \leq \frac{1}{1+t}, \quad\left\|\frac{A_{k} / 2+t}{A_{k}+t}\right\| \leq 1
$$

hold for $t \geq 0$ and lead to the trace norm estimate

$$
\left\|A_{1}^{1+s}-A_{0}^{1+s}\right\|_{1} \leq 2\left\|A_{1}-A_{0}\right\|_{1} .
$$

Since $A_{1}^{2}-A_{0}^{2}=A_{1}\left(A_{1}-A_{0}\right)+\left(A_{1}-A_{0}\right) A_{0}$, this estimate extends to $0 \leq s \leq 1$. Using the identity $A_{1}^{r+1}-A_{0}^{r+1}=A_{1}\left(A_{1}^{r}-A_{0}^{r}\right)+\left(A_{1}-A_{0}\right) A_{0}^{r}$, a simple induction yields

$$
\left\|A_{1}^{r}-A_{0}^{r}\right\|_{1} \leq(r+1)\left\|A_{1}-A_{0}\right\|_{1}
$$

for all $r \geq 1$, which proves the claim.

Step 2. For $z=w-1 \in \rho\left(h_{0}\right) \cap \rho(h)$ and any integer $q=r p \geq p$ one has

$$
(h-z)^{-q}-\left(h_{0}-z\right)^{-q}=\left(1+w(h-z)^{-1}\right)^{q} D\left(1+w\left(h_{0}-z\right)^{-1}\right)^{q},
$$

where $D=A_{1}^{r}\left(1-w A_{0}^{1 / p}\right)^{q}-\left(1-w A_{1}^{1 / p}\right)^{q} A_{0}^{r}$. Thus, to prove the first assertion of Lemma 3.1 it suffices to show that $D$ is trace class. In the expansion

$$
D=\left(A_{1}^{r}-A_{0}^{r}\right)+\sum_{m=1}^{q-1}\left(\begin{array}{c}
q \\
m
\end{array}\right)(-w)^{m}\left(A_{1}^{r} A_{0}^{r m / q}-A_{1}^{r m / q} A_{0}^{r}\right),
$$


we can use Formula (6.32) to write

$$
A_{1}^{r} A_{0}^{r m / q}-A_{1}^{r m / q} A_{0}^{r}=\frac{\sin (\pi m / q)}{\pi} \int_{0}^{\infty} \frac{A_{1}^{r}}{A_{1}^{r}+t}\left(A_{1}^{r}-A_{0}^{r}\right) \frac{A_{0}^{r}}{A_{0}^{r}+t} t^{m / q-1} \mathrm{~d} t .
$$

Proceeding as in Step 1 we get the estimate

$$
\left\|A_{1}^{r} A_{0}^{r m / q}-A_{1}^{r m / q} A_{0}^{r}\right\|_{1} \leq(1-m / q)\left\|A_{1}^{r}-A_{0}^{r}\right\|_{1},
$$

from which we conclude

$$
\|D\|_{1} \leq(1+|w|)^{q-1}\left\|A_{1}^{r}-A_{0}^{r}\right\|_{1} .
$$

Step 3. To prove the second assertion note that the second resolvent formula yields the estimate

$$
\left\|\left(A_{1}-z\right)^{-1}-\left(A_{0}-z\right)^{-1}\right\|_{1} \leq \frac{\left\|A_{1}-A_{0}\right\|_{1}}{|\operatorname{Im} z|^{2}} .
$$

The result follows from a standard application of the Helffer-Sjöstrand formula

$$
\varphi\left(A_{1}\right)-\varphi\left(A_{0}\right)=\int \bar{\partial} \widetilde{\varphi}(z)\left(\left(A_{1}-z\right)^{-1}-\left(A_{0}-z\right)^{-1}\right) \frac{\mathrm{d} z \mathrm{~d} \bar{z}}{\pi},
$$

where $\bar{\partial}=\left(\partial_{x}+\mathrm{i} \partial_{y}\right) / 2$ and $\widetilde{\varphi}$ denotes the almost-analytic extension

$$
\widetilde{\varphi}(x+\mathrm{i} y)=\left(\varphi(x)+\mathrm{i} y \varphi^{\prime}(x)+\frac{(\mathrm{i} y)^{2}}{2} \varphi^{\prime \prime}(x)\right) \chi(y),
$$

$\chi \in C_{0}^{\infty}(\mathbb{R})$ being such that $\chi(y)=1$ near $y=0$.

\subsection{Proof of Theorem 3.2}

Since the function $f(x)=(1+x)^{-p}$ is admissible, existence and completeness of the Møller operators $\Omega_{ \pm}$follow from Hypotheses (H1)-(H2) and the Kato-Rosenblum theorem (see e.g. Theorem 6.2 .5 in $[\mathrm{Y}]$ ).

1. By gauge invariance and the fact that the map $A \mapsto \omega_{0} \circ \tau^{t}(A)$ is continuous uniformly in $t$ it suffices to consider observables of the form

$$
A=a^{*}\left(f_{1}\right) \cdots a^{*}\left(f_{n}\right) a\left(g_{n}\right) \cdots a\left(g_{1}\right),
$$

with $f_{1}, \ldots, f_{n}, g_{1}, \ldots, g_{n} \in \mathfrak{h}$. For such observables, according to Equ. (2.1), one has $\omega_{0} \circ \tau^{t}(A)=\operatorname{det} M(t)$ where $M$ is the $n \times n$-matrix with entries

$$
M_{j k}(t)=\left(\mathrm{e}^{\mathrm{i} t h} g_{j}, \varrho_{0} \mathrm{e}^{\mathrm{i} t h} f_{k}\right) .
$$


Since the singular continuous spectrum of $h$ is empty one has

$$
M(t)=M^{(1)}(t)+M^{(2)}(t)+M^{(3)}(t)+M^{(4)}(t)
$$

with

$$
\begin{aligned}
M_{j k}^{(1)}(t) & =\left(\mathrm{e}^{\mathrm{i} t h} 1_{\mathrm{ac}}(h) g_{j}, \varrho_{0} \mathrm{e}^{\mathrm{i} t h} 1_{\mathrm{ac}}(h) f_{k}\right), \\
M_{j k}^{(2)}(t) & =\left(\mathrm{e}^{\mathrm{i} t h} 1_{\mathrm{ac}}(h) g_{j}, \varrho_{0} \mathrm{e}^{\mathrm{i} t h} 1_{\mathrm{pp}}(h) f_{k}\right), \\
M_{j k}^{(3)}(t) & =\left(\mathrm{e}^{\mathrm{i} t h} 1_{\mathrm{pp}}(h) g_{j}, \varrho_{0} \mathrm{e}^{\mathrm{i} t h} 1_{\mathrm{ac}}(h) f_{k}\right), \\
M_{j k}^{(4)}(t) & =\left(\mathrm{e}^{\mathrm{i} t h} 1_{\mathrm{pp}}(h) g_{j}, \varrho_{0} \mathrm{e}^{\mathrm{i} t h} 1_{\mathrm{pp}}(h) f_{k}\right) .
\end{aligned}
$$

The fact that $\varrho_{0}$ commutes with $h_{0}$ allows us to write

$$
M_{j k}^{(1)}(t)=\left(\mathrm{e}^{-\mathrm{i} t h_{0}} \mathrm{e}^{\mathrm{i} t h} 1_{\mathrm{ac}}(h) g_{j}, \varrho_{0} \mathrm{e}^{-\mathrm{i} t h_{0}} \mathrm{e}^{\mathrm{i} t h} 1_{\mathrm{ac}}(h) f_{k}\right)
$$

and from the completeness of the Møller operators we conclude that

$$
\lim _{t \rightarrow \infty} M_{j k}^{(1)}(t)=M_{j k}^{\mathrm{ac}}=\left(\Omega_{-}^{*} g_{j}, \varrho_{0} \Omega_{-}^{*} f_{k}\right) .
$$

Since $\mathfrak{h}$ is separable there exists a sequence $P_{n}$ of finite rank orthogonal projections commuting with $h$ and such that $\mathrm{s}-\lim _{n} P_{n}=1_{\mathrm{pp}}(h)$. By the Riemann-Lebesgue Lemma

$$
\lim _{t \rightarrow \infty}\left\|P_{n} \varrho_{0} \mathrm{e}^{\mathrm{i} t h} 1_{\mathrm{ac}}(h) g_{j}\right\|=0,
$$

holds for any $n, j$ and it follows from

$$
M_{j k}^{(2)}(t)=\left(\mathrm{e}^{\mathrm{i} t h} 1_{\mathrm{ac}}(h) g_{j}, \varrho_{0} \mathrm{e}^{\mathrm{i} t h}\left(I-P_{n}\right) 1_{\mathrm{pp}}(h) f_{k}\right)+\left(P_{n} \varrho_{0} \mathrm{e}^{\mathrm{i} t h} 1_{\mathrm{ac}}(h) g_{j}, \mathrm{e}^{\mathrm{i} t h} 1_{\mathrm{pp}}(h) f_{k}\right),
$$

that

$$
\lim _{t \rightarrow \infty} M^{(2)}(t)=0 .
$$

The same argument shows that

$$
\lim _{t \rightarrow \infty} M^{(3)}(t)=0,
$$

and the continuity of the determinant allows us to write

$$
\omega_{+}(A)=\lim _{T \rightarrow \infty} \frac{1}{T} \int_{0}^{T} \omega_{0} \circ \tau^{t}(A) \mathrm{d} t=\lim _{T \rightarrow \infty} \frac{1}{T} \int_{0}^{T} \operatorname{det}\left(M^{\mathrm{ac}}+M^{(4)}(t)\right) \mathrm{d} t,
$$

which exists since $M^{(4)}(t)$ and hence $\operatorname{det}\left(M^{\mathrm{ac}}+M^{(4)}(t)\right)$ are quasi-periodic functions of $t$.

2. For $f_{1}, \ldots, f_{n}, g_{1}, \ldots, g_{n} \in \mathfrak{h}_{\mathrm{ac}}(h)$ one has $M^{(2)}(t)=M^{(3)}(t)=M^{(4)}(t)=0$ and it follows from Equ. (6.34) that

$$
\lim _{t \rightarrow \infty} \omega_{0} \circ \tau^{t}(A)=\operatorname{det} M^{\mathrm{ac}}=\omega_{+}(A),
$$


is the gauge-invariant quasi-free state with density $\Omega_{-} \varrho_{0} \Omega_{-}^{*}$.

For $f \in \mathfrak{h}_{\mathrm{ac}}(h)$

$$
\gamma_{+}(a(f))=a\left(\Omega_{-}^{*} f\right),
$$

defines a $*$-isomorphism $\gamma_{+}: \operatorname{CAR}\left(\mathfrak{h}_{\mathrm{hac}}(h)\right) \rightarrow \operatorname{CAR}\left(\mathfrak{h}_{\mathrm{hc}}\left(h_{0}\right)\right)$ and it follows that

$$
\lim _{t \rightarrow \infty} \tau_{0}^{-t} \circ \tau^{t}(A)=\gamma_{+}(A),
$$

holds for all $A \in \operatorname{CAR}\left(\mathfrak{h}_{\mathrm{ac}}(h)\right)$. Since $\operatorname{Ran} \gamma_{+} \subset \operatorname{CAR}\left(\operatorname{Ran} \Omega_{-}^{*}\right)$ and $\operatorname{Ran} \Omega_{-}^{*} \subset \mathfrak{h}_{\mathrm{ac}}\left(h_{0}\right)$, Proposition 3.5 in $[\mathrm{AJPP}]$ yields the second assertion.

3. Consider $c=f(g, \cdot)$ so that $\mathrm{d} \Gamma(c)=a^{*}(f) a(g)$. Part 1 yields

$$
\begin{aligned}
\omega_{+}(\mathrm{d} \Gamma(c)) & =\lim _{T \rightarrow \infty} \frac{1}{T} \int_{0}^{T}\left(\left(\Omega_{-}^{*} g, \varrho_{0} \Omega_{-}^{*} f\right)+\sum_{\varepsilon, \varepsilon^{\prime} \in \mathrm{sp}_{\mathrm{pp}}(h)} \mathrm{e}^{\mathrm{i}\left(\varepsilon-\varepsilon^{\prime}\right) t}\left(P_{\varepsilon^{\prime}} g, \varrho_{0} P_{\varepsilon} f\right)\right) \mathrm{d} t \\
& =\left(\Omega_{-}^{*} g, \varrho_{0} \Omega_{-}^{*} f\right)+\sum_{\varepsilon \in \mathrm{sp}_{\mathrm{pp}}(h)}\left(P_{\varepsilon} g, \varrho_{0} P_{\varepsilon} f\right) \\
& =\operatorname{Tr}\left(\varrho_{+} c\right) .
\end{aligned}
$$

The result follows from a density argument.

\subsection{Proof of Theorem 4.1}

Our proof is based on stationary representations of the Møller operators and of the corresponding scattering matrix. For the reader convenience we briefly recall some basic facts from the stationary scattering theory of trace class perturbations. We refer to $[\mathrm{Y}]$ for a detailed exposition. Set $\ell_{0}=f_{\eta}\left(h_{0}\right)$ and $\ell=f_{\eta}(h)$. Then $\ell_{0}$ and $\ell$ are two self-adjoint operators such that $v=\ell-\ell_{0}$ is trace class. By the Kato-Rosenblum theorem the Møller operators

$$
W_{ \pm}=\mathrm{s}-\lim _{t \rightarrow \pm \infty} \mathrm{e}^{\mathrm{i} t \ell} \mathrm{e}^{-\mathrm{i} t \ell_{0}} 1_{\mathrm{ac}}\left(\ell_{0}\right),
$$

exist and are complete. In particular the scattering matrix $\widetilde{S}=W_{+}^{*} W_{-}$is unitary on $\mathfrak{h}_{\text {ac }}\left(\ell_{0}\right)$.

Set $E_{+}=\left[0,(p \eta)^{-1}\left[\right.\right.$ and $\left.E_{-}=\right](p \eta)^{-1}, \infty\left[\right.$ and note that $f_{\eta}$ satisfies $f_{\eta}^{\prime}(x)>0$ for $x \in E_{+}$and $f_{\eta}^{\prime}(x)<0$ for $x \in E_{-}$. Since $f_{\eta}$ is admissible the invariance principle for Møller operators (Theorem 6.2.5 in [Y]) yields

$$
\Omega_{ \pm}=W_{ \pm} 1_{E_{+}}\left(h_{0}\right)+W_{\mp} 1_{E_{-}}\left(h_{0}\right) .
$$

Denote the two inverse branches of $f_{\eta}$ by $g_{\eta}^{ \pm}=\left.f_{\eta}\right|_{E \pm}{ }^{-1}$. Then $\mathfrak{h}_{\mathrm{ac}}\left(h_{0}\right)=\mathfrak{h}_{\mathrm{ac}}\left(\ell_{0}\right)$ has a direct integral decomposition

$$
V: \mathfrak{h}_{\mathrm{ac}}\left(\ell_{0}\right) \rightarrow \int_{\mathrm{sp}_{\mathrm{ac}}\left(\ell_{0}\right)}^{\oplus} \tilde{\mathfrak{h}}(\lambda) \mathrm{d} \lambda,
$$


associated to $\ell_{0}$ and related to the decomposition $(4.9)$ by $\tilde{\mathfrak{h}}(\lambda)=\mathfrak{h}\left(g_{\eta}^{+}(\lambda)\right) \oplus \mathfrak{h}\left(g_{\eta}^{-}(\lambda)\right)$ and

$$
(V u)(\lambda)=\left|g_{\eta}^{+^{\prime}}(\lambda)\right|^{1 / 2}(U u)\left(g_{\eta}^{+}(\lambda)\right) \oplus\left|g_{\eta}^{-\prime}(\lambda)\right|^{1 / 2}(U u)\left(g_{\eta}^{-}(\lambda)\right) .
$$

According to Equ. (6.36) the corresponding fibers of scattering matrices are related by

$$
\widetilde{S}(\lambda)=S\left(g_{\eta}^{+}(\lambda)\right) \oplus S\left(g_{\eta}^{-}(\lambda)\right)^{*}
$$

Since $v$ is trace class it has a factorization $v=x^{*} y$ where $x, y: \mathfrak{h} \rightarrow \mathfrak{K}$ are HilbertSchmidt. This leads, for the resolvents $r_{0}(z)=\left(\ell_{0}-z\right)^{-1}$ and $r(z)=(\ell-z)^{-1}$, to the identities

$$
\begin{aligned}
r(z) & =r_{0}(z)-r_{0}(z) x^{*} Q(z) y r_{0}(z) \\
& =r_{0}(z)-r_{0}(z) y^{*} Q(\bar{z})^{*} x r_{0}(z)
\end{aligned}
$$

where $Q(z)=\left(1+y r_{0}(z) x^{*}\right)^{-1}$ and $Q(\bar{z})^{*}=\left(1+x r_{0}(z) y^{*}\right)^{-1}$.

If $a$ and $b$ are Hilbert-Schmidt operators from $\mathfrak{h}$ to some arbitrary Hilbert spaces then they are $\ell_{0^{-}}$and $\ell$-smooth. The boundary values

$$
a r_{0}(\lambda \pm \mathrm{i} 0) b^{*}=\lim _{\eta \downarrow 0} a r_{0}(\lambda \pm \mathrm{i} \eta) b^{*}
$$

as well as those obtained by replacing $r_{0}$ by $r$ exist in the Hilbert-Schmidt norm for Lebesgue almost all $\lambda \in \mathbb{R}$ (Corollary 6.1.10 in [Y]). This applies in particular to the operators $y r_{0}(\lambda \pm \mathrm{i} 0) x^{*}$ and $y r(\lambda \pm \mathrm{i} 0) x^{*}$ and it follows that

$$
Q(\lambda \pm \mathrm{i} 0)=\left(1+y r_{0}(\lambda \pm \mathrm{i} 0) x^{*}\right)^{-1}=1-y r(\lambda \pm \mathrm{i} 0) x^{*},
$$

exist for Lebesgue almost all $\lambda$.

If $a$ is a Hilbert-Schmidt operator from $\mathfrak{h}_{\mathrm{ac}}\left(\ell_{0}\right)$ to some Hilbert space $\mathfrak{K}$ then the formula

$$
\mathfrak{K} \ni u \mapsto Z(a, \lambda) u=\left(V a^{*} u\right)(\lambda) \in \mathfrak{h}(\lambda),
$$

defines, for almost every $\lambda \in \operatorname{sp}_{\text {ac }}\left(\ell_{0}\right)$, a Hilbert-Schmidt operator $Z(a, \lambda): \mathfrak{K} \rightarrow \tilde{\mathfrak{h}}(\lambda)$. For any tempered conserved charge $q$, Equ. (6.37) yields

$$
Z(a q, \lambda)=\widetilde{q}(\lambda) Z(a, \lambda)=\left(q\left(g_{\eta}^{+}(\lambda)\right) \oplus q\left(g_{\eta}^{-}(\lambda)\right)\right) Z(a, \lambda) .
$$

Moreover, the relation

$$
Z(a, \lambda)^{*} Z(b, \lambda)=\frac{1}{2 \pi \mathrm{i}} a\left(r_{0}(\lambda+\mathrm{i} 0)-r_{0}(\lambda-\mathrm{i} 0)\right) b^{*},
$$

holds for any Hilbert-Schmidt $a, b$ (see e.g. Sections 5.4 and 7.5 in $[Y]$ ). 
Using the resolvent identities (6.39) and (6.40), the Møller operators $W_{ \pm}$can be expressed by the Abelian limit

$$
\begin{aligned}
\left(V W_{ \pm}^{*} a^{*} u\right)(\lambda) & =\lim _{\delta \downarrow 0} \delta \int_{0}^{\infty} \mathrm{e}^{-\delta t}\left(V \mathrm{e}^{ \pm \mathrm{i} t \ell_{0}} \mathrm{e}^{\mp \mathrm{i} t \ell} a^{*} u\right)(\lambda) \mathrm{d} t \\
& =\lim _{\delta \downarrow 0} \delta \int_{0}^{\infty}\left(V \mathrm{e}^{\mp \mathrm{i} t(\ell-\lambda \mp \mathrm{i} \delta)} a^{*} u\right)(\lambda) \mathrm{d} t \\
& =\lim _{\delta \downarrow 0} \mp \mathrm{i} \delta\left(V r(\lambda \pm \mathrm{i} \delta) a^{*} u\right)(\lambda) \\
& =\left(V a^{*} u\right)(\lambda)-\left(V x^{*} Q(\lambda \pm \mathrm{i} 0) y r_{0}(\lambda \pm \mathrm{i} 0) a^{*} u\right)(\lambda) \\
& =\left(V a^{*} u\right)(\lambda)-\left(V y^{*} Q(\lambda \mp \mathrm{i} 0)^{*} x r_{0}(\lambda \pm \mathrm{i} 0) a^{*} u\right)(\lambda)
\end{aligned}
$$

This formal calculation can be justified (see Theorem 5.6.1 in [Y]) and leads to the formulas

$$
\begin{aligned}
Z\left(a W_{ \pm}, \lambda\right) & =Z(a, \lambda)-Z(x, \lambda) Q(\lambda \pm \mathrm{i} 0) y r_{0}(\lambda \pm \mathrm{i} 0) a^{*} \\
Z\left(a W_{ \pm}, \lambda\right)^{*} & =Z(a, \lambda)^{*}-a r_{0}(\lambda \mp \mathrm{i} 0) x^{*} Q(\lambda \mp \mathrm{i} 0) Z(y, \lambda)^{*} .
\end{aligned}
$$

The scattering matrix $V W_{+}^{*} W_{-} V^{*}$ acts on each fiber $\tilde{\mathfrak{h}}(\lambda)$ by multiplication with the unitary operator (see Section 5.7 in $[\mathrm{Y}]$ )

$$
\begin{aligned}
\tilde{S}(\lambda) & =I-2 \pi \mathrm{i} Z(x, \lambda) Q(\lambda+\mathrm{i} 0) Z(y, \lambda)^{*} \\
& =I-2 \pi \mathrm{i} Z(y, \lambda) Q(\lambda-\mathrm{i} 0)^{*} Z(x, \lambda)^{*}
\end{aligned}
$$

We now start with the regularized flux $\Phi_{q^{(\Lambda)}}^{\eta}$ for which Theorem 3.2 and Proposition 3.5 gives

$$
\omega_{+}\left(\Phi_{q^{(\Lambda)}}^{\eta}\right)=\operatorname{Tr}\left(\varrho_{0} \Omega_{-}^{*} \varphi_{q^{(\Lambda)}}^{\eta} \Omega_{-}\right)=\operatorname{Tr}\left(\left(V \varrho_{0} V^{*}\right) V \Omega_{-}^{*} \varphi_{q^{(\Lambda)}}^{\eta} \Omega_{-} V^{*}\right)
$$

Using the factorization $f_{\eta}(h)-f_{\eta}\left(h_{0}\right)=x^{*} y$ in Equ. (3.5) and inserting the resulting identity

$$
\varphi_{q^{(\Lambda)}}^{\eta}=\mathrm{i}\left(\left(x q^{(\Lambda)}\right)^{*} y-x^{*}\left(y q^{(\Lambda)}\right)\right)
$$

into Equ. (6.46) we obtain, taking Equ. (6.36) into account

$$
\omega_{+}\left(\Phi_{q^{(\Lambda)}}^{\eta}\right)=\int_{\mathrm{sp}_{\mathrm{ac}}\left(\ell_{0}\right)} \operatorname{Tr}_{\tilde{\mathfrak{h}}(\lambda)}\left(\left[\varrho_{0}\left(g_{\eta}^{+}(\lambda)\right) \oplus \varrho_{0}\left(g_{\eta}^{-}(\lambda)\right)\right]\left[P_{+} D_{+}(\lambda)+P_{-} D_{-}(\lambda)\right]\right) \mathrm{d} \lambda,
$$

where

$$
D_{ \pm}(\lambda)=\mathrm{i}\left(Z\left(x q^{(\Lambda)} W_{\mp}, \lambda\right) Z\left(y W_{\mp}, \lambda\right)^{*}-Z\left(x W_{\mp}, \lambda\right) Z\left(y q^{(\Lambda)} W_{\mp}, \lambda\right)^{*}\right),
$$

and $P_{ \pm}$projects on the first/second component of $\tilde{\mathfrak{h}}(\lambda)$. 
Identities (6.43) and (6.41) yield

$$
\begin{aligned}
Z\left(x W_{ \pm}, \lambda\right) & =Z(x, \lambda)\left(I-Q(\lambda \pm \mathrm{i} 0) y r_{0}(\lambda \pm \mathrm{i} 0) x^{*}\right)=Z(x, \lambda) Q(\lambda \pm \mathrm{i} 0), \\
Z\left(x q^{(\Lambda)} W_{ \pm}, \lambda\right) & =\widetilde{q}^{(\Lambda)}(\lambda) Z(x, \lambda)-Z(x, \lambda) Q(\lambda \pm \mathrm{i} 0) y r_{0}(\lambda \pm \mathrm{i} 0) q^{(\Lambda)} x^{*},
\end{aligned}
$$

while (6.44) and (6.41) yield

$$
\begin{aligned}
Z\left(y W_{ \pm}, \lambda\right)^{*} & =\left(I-y r_{0}(\lambda \mp \mathrm{i} 0) x^{*} Q(\lambda \mp \mathrm{i} 0)\right) Z(y, \lambda)^{*}=Q(\lambda \mp \mathrm{i} 0) Z(y, \lambda)^{*} \\
Z\left(y q^{(\Lambda)} W_{ \pm}, \lambda\right)^{*} & =Z(y, \lambda)^{*} \widetilde{q}^{(\Lambda)}(\lambda)-y q^{(\Lambda)} r_{0}(\lambda \mp \mathrm{i} 0) x^{*} Q(\lambda \mp \mathrm{i} 0) Z(y, \lambda)^{*} .
\end{aligned}
$$

Inserting these four relations into Equ. (6.48) further gives

$$
\begin{aligned}
D_{ \pm}(\lambda) & =\mathrm{i}\left(\widetilde{q}^{(\Lambda)}(\lambda) Z(x, \lambda) Q(\lambda \pm \mathrm{i} 0) Z(y, \lambda)^{*}-Z(x, \lambda) Q(\lambda \mp \mathrm{i} 0) Z(y, \lambda)^{*} \widetilde{q}^{(\Lambda)}(\lambda)\right. \\
& \left.+Z(x, \lambda) Q(\lambda \mp \mathrm{i} 0)\left[y q^{(\Lambda)}\left(r_{0}(\lambda \pm \mathrm{i} 0)-r_{0}(\lambda \mp \mathrm{i} 0)\right) x^{*}\right] Q(\lambda \pm \mathrm{i} 0) Z(y, \lambda)^{*}\right) .
\end{aligned}
$$

From Equ. (6.42) and (6.41) we get

$$
\begin{aligned}
y q^{(\Lambda)}\left(r_{0}(\lambda \pm \mathrm{i} 0)-r_{0}(\lambda \mp \mathrm{i} 0)\right) x^{*} & = \pm 2 \pi \mathrm{i} Z\left(y q^{(\Lambda)}, \lambda\right)^{*} Z(x, \lambda) \\
& = \pm 2 \pi \mathrm{i} Z(y, \lambda)^{*} \widetilde{q}^{(\Lambda)}(\lambda) Z(x, \lambda)
\end{aligned}
$$

and thus, using Formulae (6.45), we obtain

$$
D_{+}(\lambda)=\frac{1}{2 \pi}\left(\widetilde{q}^{(\Lambda)}(\lambda)-\widetilde{S}(\lambda)^{*} q^{(\Lambda)}(\lambda) \widetilde{S}(\lambda)\right)
$$

and

$$
D_{-}(\lambda)=\frac{1}{2 \pi}\left(\widetilde{S}(\lambda) q^{(\Lambda)}(\lambda) \widetilde{S}(\lambda)^{*}-\widetilde{q}^{(\Lambda)}(\lambda)\right) .
$$

The $S$-matrix relation (6.38) then leads to

$$
\begin{aligned}
P_{+} D_{+}(\lambda)+P_{-} D_{-}(\lambda)=\frac{1}{2 \pi} \quad\left[\left(q^{(\Lambda)}\left(g_{\eta}^{+}(\lambda)\right)-S\left(g_{\eta}^{+}(\lambda)\right)^{*} q^{(\Lambda)}\left(g_{\eta}^{+}(\lambda)\right) S\left(g_{\eta}^{+}(\lambda)\right)\right)\right. \\
\left.\oplus \quad\left(S\left(g_{\eta}^{-}(\lambda)\right)^{*} q^{(\Lambda)}\left(g_{\eta}^{-}(\lambda)\right) S\left(g_{\eta}^{-}(\lambda)\right)-q^{(\Lambda)}\left(g_{\eta}^{-}(\lambda)\right)\right)\right]
\end{aligned}
$$

which, upon insertion into Equ. (6.47) and after a change of the integration variable, yields

$$
\begin{aligned}
\omega_{+}\left(\Phi_{q^{(\Lambda)}}^{\eta}\right) & =\int_{E_{+}} \operatorname{Tr}_{\mathfrak{h}(\varepsilon)}\left(\varrho_{0}(\varepsilon)\left[q^{(\Lambda)}(\varepsilon)-S(\varepsilon)^{*} q^{(\Lambda)}(\varepsilon) S(\varepsilon)\right]\right)\left|f_{\eta}^{\prime}(\varepsilon)\right| \frac{\mathrm{d} \varepsilon}{2 \pi} \\
& -\int_{E_{-}} \operatorname{Tr}_{\mathfrak{h}(\varepsilon)}\left(\varrho_{0}(\varepsilon)\left[q^{(\Lambda)}(\varepsilon)-S(\varepsilon)^{*} q^{(\Lambda)}(\varepsilon) S(\varepsilon)\right]\right)\left|f_{\eta}^{\prime}(\varepsilon)\right| \frac{\mathrm{d} \varepsilon}{2 \pi}
\end{aligned}
$$

Recall that $q^{(\Lambda)}(\cdot)$ is supported by $[0, \Lambda]$ and that $\left.E_{-}=\right](\mathrm{p} \eta)^{-1}, \infty[$. Thus, for sufficiently small $\eta$, one has $E_{-} \cap[0, \Lambda]=\emptyset$ and the second integral on the right hand side of the 
last formula vanishes. From the fact that the function $f(\varepsilon)=(1+\varepsilon)^{-p}$ is admissible it immediately follows from the estimate (Theorem 7.6.6 in $[\mathrm{Y}]$ )

$$
\int_{\mathrm{sp}_{\mathrm{ac}}\left(h_{0}\right)}\|S(\varepsilon)-I\|_{1}\left|f^{\prime}(\varepsilon)\right| \frac{\mathrm{d} \varepsilon}{2 \pi} \leq\left\|f(h)-f\left(h_{0}\right)\right\|_{1}=C<\infty
$$

that

$$
\int_{\mathrm{sp}_{\mathrm{ac}}\left(h_{0}\right) \cap[0, \Lambda]}\|S(\varepsilon)-I\|_{1} \frac{\mathrm{d} \varepsilon}{2 \pi} \leq \frac{C}{p}(1+\Lambda)^{p+1} .
$$

Since

$$
\left|\operatorname{Tr}_{\mathfrak{h}(\varepsilon)}\left(\varrho_{0}(\varepsilon)\left[q^{(\Lambda)}(\varepsilon)-S(\varepsilon)^{*} q^{(\Lambda)}(\varepsilon) S(\varepsilon)\right]\right)\right| \leq 4\left\|\varrho_{0}(\varepsilon)\right\|\|q(\varepsilon)\|\|S(\varepsilon)-I\|_{1},
$$

Lebesgue dominated convergence theorem allows us to conclude that

$$
\begin{aligned}
\omega_{+}\left(\Phi_{q^{(\Lambda)}}\right)=\lim _{\eta \rightarrow 0} \omega_{+}\left(\Phi_{q^{(\Lambda)}}^{\eta}\right) & =\int_{\mathrm{sp}_{\mathrm{ac}}\left(h_{0}\right)} \operatorname{Tr}_{\mathfrak{h}(\varepsilon)}\left(\varrho_{0}(\varepsilon)\left[q^{(\Lambda)}(\varepsilon)-S(\varepsilon)^{*} q^{(\Lambda)}(\varepsilon) S(\varepsilon)\right]\right) \frac{\mathrm{d} \varepsilon}{2 \pi} \\
& =\int_{\mathrm{sp}_{\mathrm{ac}}\left(h_{0}\right) \cap[0, \Lambda]} \operatorname{Tr}_{\mathfrak{h}(\varepsilon)}\left(\varrho_{0}(\varepsilon)\left[q(\varepsilon)-S(\varepsilon)^{*} q(\varepsilon) S(\varepsilon)\right]\right) \frac{\mathrm{d} \varepsilon}{2 \pi} .
\end{aligned}
$$

Finally, under condition (4.10), the estimate (6.49) and (6.50) show that

$$
\omega_{+}\left(\Phi_{q}\right)=\lim _{\Lambda \rightarrow \infty} \omega_{+}\left(\Phi_{q^{(\Lambda)}}\right)=\int_{\operatorname{sp}_{\mathrm{ac}}\left(h_{0}\right)} \operatorname{Tr}_{\mathfrak{h}(\varepsilon)}\left(\varrho_{0}(\varepsilon)\left[q(\varepsilon)-S(\varepsilon)^{*} q(\varepsilon) S(\varepsilon)\right]\right) \frac{\mathrm{d} \varepsilon}{2 \pi}
$$

\subsection{Proof of Proposition 4.4}

Using Definition (4.26) we rewrite Formula (4.22) as

$$
\operatorname{Ep}\left(\omega_{+}\right)=\sum_{k j} \int \xi_{k}(\varepsilon) F\left(\xi_{j}(\varepsilon)\right) D_{k j}(\varepsilon) \frac{\mathrm{d} \varepsilon}{2 \pi} .
$$

Since we will estimate the integrand on the right hand side of this formula for fixed $\varepsilon$ we omit it. First note that, by Equ. (4.17) (a consequence of the unitarity of the scattering matrix) one has

$$
\sum_{k} D_{k j}=\sum_{j} D_{k j}=0
$$

We also recall that

$$
D_{k j} \geq 0 \quad \text { for } \quad k \neq j .
$$

For fixed $\xi_{1}, \cdots, \xi_{M} \in \mathbb{R}$ we consider the sum

$$
\mathfrak{S} \equiv \sum_{k j} D_{k j} \xi_{k} F\left(\xi_{j}\right)
$$


Let $\pi$ be a permutation such that

$$
\xi_{\pi(1)} \leq \xi_{\pi(2)} \leq \cdots \leq \xi_{\pi(M)}
$$

We can write

$$
\mathfrak{S}=\sum_{k j} \tilde{D}_{k j} \tilde{\xi}_{k} F\left(\tilde{\xi}_{j}\right)
$$

where $\tilde{\xi}_{i} \equiv \xi_{\pi(i)}$ and the matrix $\tilde{D}_{k j} \equiv D_{\pi(k) \pi(j)}$ also satisfies (6.51) and (6.52). In particular, from Equ. (6.51) it follows that

$$
\begin{aligned}
\sum_{k} \tilde{D}_{k j} \tilde{\xi}_{k} & =\sum_{k} \tilde{D}_{k j}\left(\tilde{\xi}_{k}-\tilde{\xi}_{1}\right)=\sum_{k} \tilde{D}_{k j} \sum_{l<k}\left(\tilde{\xi}_{l+1}-\tilde{\xi}_{l}\right) \\
& =\sum_{l}\left(\sum_{k>l} \tilde{D}_{k j}\right)\left(\tilde{\xi}_{l+1}-\tilde{\xi}_{l}\right)=\sum_{l} C_{l j}\left(\tilde{\xi}_{l+1}-\tilde{\xi}_{l}\right) .
\end{aligned}
$$

Since Equ. (6.51) allows to rewrite the matrix $C$ as

$$
C_{l j}=\left\{\begin{array}{cl}
\sum_{k>l} \tilde{D}_{k j} & \text { for } \quad l \geq j, \\
-\sum_{k \leq l} \tilde{D}_{k j} & \text { for } \quad l<j,
\end{array}\right.
$$

it follows from Equ. (6.52) that $C_{l j} \geq 0$ for $l \geq j$ and $C_{l j} \leq 0$ otherwise. Rewriting $\mathfrak{S}$ as

$$
\mathfrak{S}=\sum_{l<j} C_{l j}\left(\tilde{\xi}_{l+1}-\tilde{\xi}_{l}\right) F\left(\tilde{\xi}_{j}\right)+\sum_{l \geq j} C_{l j}\left(\tilde{\xi}_{l+1}-\tilde{\xi}_{l}\right) F\left(\tilde{\xi}_{j}\right),
$$

and using the facts that $\tilde{\xi}_{l+1}-\tilde{\xi}_{l} \geq 0$ and $F\left(\tilde{\xi}_{j}\right) \leq F\left(\tilde{\xi}_{l+1}\right)$ in the first sum while $F\left(\tilde{\xi}_{l}\right) \leq$ $F\left(\tilde{\xi}_{j}\right)$ in the second we obtain

$$
\mathfrak{S} \geq \sum_{l}\left(\sum_{j>l} C_{l j} F\left(\tilde{\xi}_{l+1}\right)+\sum_{j \leq l} C_{l j} F\left(\tilde{\xi}_{l}\right)\right)\left(\tilde{\xi}_{l+1}-\tilde{\xi}_{l}\right) .
$$

Since $\sum_{j} C_{l j}=\sum_{j} \sum_{k>l} \tilde{D}_{k j}=0$, this is the same as

$$
\mathfrak{S} \geq \sum_{l}\left(\sum_{j \leq l} C_{l j} F\left(\tilde{\xi}_{l}\right)-\sum_{j \leq l} C_{l j} F\left(\tilde{\xi}_{l+1}\right)\right)\left(\tilde{\xi}_{l+1}-\tilde{\xi}_{l}\right),
$$

and we obtain

$$
\mathfrak{S} \geq \sum_{l}\left(F\left(\tilde{\xi}_{l}\right)-F\left(\tilde{\xi}_{l+1}\right)\right)\left(\tilde{\xi}_{l+1}-\tilde{\xi}_{l}\right) \sum_{j \leq l} C_{l j} \geq 0 .
$$


We further note that, by Equ. (6.51),

$$
\begin{aligned}
B_{l} & =\sum_{j \leq l} C_{l j}=\sum_{j \leq l} \sum_{k>l} \tilde{D}_{k j} \\
& =\sum_{j \leq l}\left(-\sum_{k \leq l} \tilde{D}_{k j}\right)=\sum_{j>l} \sum_{k \leq l} \tilde{D}_{k j},
\end{aligned}
$$

from which it follows that, if $m \leq l<n$, one has

$$
B_{l} \geq \tilde{D}_{m n}, \quad \text { and } \quad B_{l} \geq \tilde{D}_{n m} .
$$

Given a pair $(m, n)$, with $m \neq n$, let us set $m^{\prime} \equiv \min \left(\pi^{-1}(m), \pi^{-1}(n)\right)$ and $n^{\prime} \equiv$ $\max \left(\pi^{-1}(m), \pi^{-1}(n)\right)$. From the estimate (6.53), we get

$$
\mathfrak{S} \geq \sum_{m^{\prime} \leq l<n^{\prime}}\left(F\left(\tilde{\xi}_{l}\right)-F\left(\tilde{\xi}_{l+1}\right)\right)\left(\tilde{\xi}_{l+1}-\tilde{\xi}_{l}\right) B_{l}
$$

and from Equ. (6.54) we conclude

$$
\mathfrak{S} \geq D_{m n} \sum_{m^{\prime} \leq l<n^{\prime}}\left(F\left(\tilde{\xi}_{l}\right)-F\left(\tilde{\xi}_{l+1}\right)\right)\left(\tilde{\xi}_{l+1}-\tilde{\xi}_{l}\right) .
$$

The remaining sum is easily estimated, using the fact that $F^{\prime}(x)=-F(x) F(-x)$ and Jensen inequality

$$
\begin{aligned}
\sum_{m^{\prime} \leq l<n^{\prime}}\left(F\left(\tilde{\xi}_{l}\right)-F\left(\tilde{\xi}_{l+1}\right)\right)\left(\tilde{\xi}_{l+1}-\tilde{\xi}_{l}\right) & \geq \min _{x \in\left[\tilde{\xi}_{m^{\prime}}, \tilde{\xi}_{n^{\prime}}\right]}\left(-F^{\prime}(x)\right) \sum_{m^{\prime} \leq l<n^{\prime}}\left(\tilde{\xi}_{l+1}-\tilde{\xi}_{l}\right)^{2} \\
& \geq F\left(\left|\tilde{\xi}_{n^{\prime}}\right|\right) F\left(\left|\tilde{\xi}_{m^{\prime}}\right|\right) \frac{1}{n^{\prime}-m^{\prime}}\left(\tilde{\xi}_{n^{\prime}}-\tilde{\xi}_{m^{\prime}}\right)^{2} \\
& \geq F\left(\left|\xi_{n}\right|\right) F\left(\left|\xi_{m}\right|\right) \frac{1}{M}\left(\xi_{n}-\xi_{m}\right)^{2} .
\end{aligned}
$$

Inserting the resulting estimate

$$
\mathfrak{S}=\sum_{k j} D_{k j} \xi_{k} F\left(\xi_{j}\right) \geq \frac{1}{M} D_{m n} F\left(\left|\xi_{n}\right|\right) F\left(\left|\xi_{m}\right|\right)\left(\xi_{n}-\xi_{m}\right)^{2},
$$

into Equ. (4.22) leads to the desired inequality.

\subsection{Proof of Proposition 5.1}

We start with the formula of Proposition 3.5

$$
\omega_{X+}\left(\Phi_{k}^{\mathrm{u}}\right)=\operatorname{Tr}\left(\Omega_{-} \varrho_{0}(X) \Omega_{-}^{*} \varphi_{k}^{\mathrm{u}}\right)
$$


where

$$
\varrho_{0}(X)=\bigoplus_{k=1}^{M}\left(1+\mathrm{e}^{\left(\beta-X_{k}^{\mathrm{h}}\right) h_{k}-\left(\beta \mu+X_{k}^{\mathrm{c}}\right) 1_{k}}\right)^{-1} .
$$

Since $\varphi_{k}^{\mathrm{u}}$ is trace class and

$$
\left.\partial_{X_{j}^{\mathrm{v}}} \varrho_{0}(X)\right|_{X=0}=f_{\mathrm{eq}}\left(h_{0}\right)\left(1-f_{\mathrm{eq}}\left(h_{0}\right)\right) h_{0}^{n_{\mathrm{v}}} 1_{j},
$$

in the norm topology of $\mathcal{B}(\mathfrak{h})$ we obtain, using the intertwining property of the Møller operators,

$$
L_{k j}^{\mathrm{uv}}=\operatorname{Tr}\left(f_{\mathrm{eq}}(h)\left(1-f_{\mathrm{eq}}(h)\right) h^{n_{\mathrm{v}}} P_{j}^{-} \varphi_{k}^{\mathrm{u}}\right),
$$

where $P_{j}^{-}=\Omega_{-} 1_{j} \Omega_{-}^{*}$. For $\Lambda>0$ let us set $h_{0}^{(\Lambda)}=h_{0} 1_{]-\infty, \Lambda]}\left(h_{0}\right), h^{(\Lambda)}=h 1_{]-\infty, \Lambda]}(h)$ and $\varphi_{k}^{\mathrm{u}(\Lambda)}=\mathrm{i}\left[h_{0}^{(\Lambda)} 1_{k}, h\right]$. Since

$$
\lim _{\Lambda \rightarrow \infty} f_{\text {eq }}(h) h^{(\Lambda)}=f_{\text {eq }}(h) h,
$$

in the norm topology of $\mathcal{B}(\mathfrak{h})$ and

$$
\lim _{\Lambda \rightarrow \infty} \varphi_{k}^{\mathrm{u}(\Lambda)}=\varphi_{k}^{\mathrm{u}}
$$

in the trace class topology we have

$$
L_{k j}^{\mathrm{uv}}=\lim _{\Lambda \rightarrow \infty} L_{k j}^{\mathrm{uv}(\Lambda)}
$$

where

$$
L_{k j}^{\mathrm{uv}(\Lambda)}=\operatorname{Tr}\left(f_{\mathrm{eq}}(h)\left(1-f_{\mathrm{eq}}(h)\right) h^{(\Lambda) n_{\mathrm{v}}} P_{j}^{-} \varphi_{k}^{\mathrm{u}(\Lambda)}\right) .
$$

Since

$$
\mathrm{i} \partial_{\theta}\left(1-f_{\text {eq }}(h)\right) \mathrm{e}^{-\theta h} h_{0}^{(\Lambda) n_{\mathrm{u}}} 1_{k} \mathrm{e}^{\theta h} f_{\text {eq }}(h)=\left(1-f_{\text {eq }}(h)\right) \mathrm{e}^{-\theta h} \varphi_{k}^{\mathrm{u}(\Lambda)} \mathrm{e}^{\theta h} f_{\text {eq }}(h),
$$

where the right hand side is a continuous function of $\theta \in[0, \beta]$ in the trace class topology, we have

$$
\int_{0}^{\beta}\left(1-f_{\text {eq }}(h)\right) \mathrm{e}^{-\theta h} \varphi_{k}^{\mathrm{u}(\Lambda)} \mathrm{e}^{\theta h} f_{\text {eq }}(h) \mathrm{d} \theta=\mathrm{i}\left[f_{\text {eq }}(h), h_{0}^{(\Lambda) n_{\mathrm{u}}} 1_{k}\right],
$$

where the integral is a Riemann integral in the trace class topology. From the fact that the asymptotic projection $P_{j}^{-}$commutes with $h$ we conclude that

$$
L_{k j}^{\mathrm{uv}(\Lambda)}=\operatorname{Tr}\left(h^{(\Lambda) n_{\mathrm{v}}} P_{j}^{-}\left(1-f_{\mathrm{eq}}(h)\right) \mathrm{e}^{-\theta h} \varphi_{k}^{\mathrm{u}(\Lambda)} \mathrm{e}^{\theta h} f_{\text {eq }}(h)\right),
$$


holds for $\theta \in[0, \beta]$. Therefore, we can also write

$$
\begin{aligned}
L_{k j}^{\mathrm{uv}(\Lambda)} & =\frac{1}{\beta} \int_{0}^{\beta} \mathrm{d} \theta \operatorname{Tr}\left(h^{(\Lambda) n_{\mathrm{v}}} P_{j}^{-}\left(1-f_{\text {eq }}(h)\right) \mathrm{e}^{-\theta h} \varphi_{k}^{\mathrm{u}(\Lambda)} \mathrm{e}^{\theta h} f_{\text {eq }}(h)\right) \\
& =\frac{1}{\beta} \operatorname{Tr}\left(h^{(\Lambda) n_{\mathrm{v}}} P_{j}^{-} \mathrm{i}\left[f_{\text {eq }}(h), h_{0}^{(\Lambda) n_{\mathrm{u}}} 1_{k}\right]\right) \\
& =\frac{1}{\beta} \operatorname{Tr}\left(h^{(\Lambda) n_{\mathrm{v}}} P_{j}^{-} \mathrm{i}\left[f_{\text {eq }}(h)-f_{\text {eq }}\left(h_{0}\right), h_{0}^{(\Lambda) n_{\mathrm{u}}} 1_{k}\right]\right) \\
& =\frac{\mathrm{i}}{\beta} \operatorname{Tr}\left(P_{j}^{-} h^{(\Lambda) n_{\mathrm{v}}}\left(f_{\text {eq }}(h)-f_{\text {eq }}\left(h_{0}\right)\right) h_{0}^{(\Lambda) n_{\mathrm{u}}} 1_{k}-1_{k} h_{0}^{(\Lambda) n_{\mathrm{v}}}\left(f_{\text {eq }}(h)-f_{\text {eq }}\left(h_{0}\right)\right) h^{(\Lambda) n_{\mathrm{u}}} P_{j}^{-}\right) .
\end{aligned}
$$

Set $\left\langle h_{0}\right\rangle=1+h_{0}$ and $\langle h\rangle=1+h$. From the identity

$$
\begin{aligned}
C= & \langle h\rangle^{2}\left(f_{\text {eq }}(h)-f_{\text {eq }}\left(h_{0}\right)\right)\left\langle h_{0}\right\rangle^{2} \\
= & \langle h\rangle^{4} f_{\text {eq }}(h)-\left\langle h_{0}\right\rangle^{4} f_{\text {eq }}\left(h_{0}\right)-\langle h\rangle^{3} f_{\text {eq }}(h) v-v f_{\text {eq }}\left(h_{0}\right)\left\langle h_{0}\right\rangle^{3} \\
& -\langle h\rangle f_{\text {eq }}\left(h_{0}\right) v\left\langle h_{0}\right\rangle-\langle h\rangle v f_{\text {eq }}(h)\left\langle h_{0}\right\rangle,
\end{aligned}
$$

we deduce that $C$ is trace class. Writing

$$
h^{(\Lambda) n_{\mathrm{v}}}\left(f_{\mathrm{eq}}(h)-f_{\mathrm{eq}}\left(h_{0}\right)\right) h_{0}^{(\Lambda) n_{\mathrm{u}}}=h^{(\Lambda) n_{\mathrm{v}}}\langle h\rangle^{-2} C\left\langle h_{0}\right\rangle^{-2} h_{0}^{(\Lambda) n_{\mathrm{v}}},
$$

we conclude that

$$
\lim _{\Lambda \rightarrow \infty} h^{(\Lambda) n_{\mathrm{v}}}\left(f_{\mathrm{eq}}(h)-f_{\mathrm{eq}}\left(h_{0}\right)\right) h_{0}^{(\Lambda) n_{\mathrm{u}}}=h^{n_{\mathrm{v}}}\left(f_{\mathrm{eq}}(h)-f_{\mathrm{eq}}\left(h_{0}\right)\right) h_{0}^{n_{\mathrm{u}}},
$$

holds in trace class topology. Hence we obtain

$$
\begin{aligned}
L_{k j}^{\mathrm{uv}} & =\frac{\mathrm{i}}{\beta} \operatorname{Tr}\left(P_{j}^{-} h^{n_{\mathrm{v}}}\left(f_{\mathrm{eq}}(h)-f_{\mathrm{eq}}\left(h_{0}\right)\right) h_{0}^{n_{\mathrm{u}}} 1_{k}-1_{k} h_{0}^{n_{\mathrm{v}}}\left(f_{\mathrm{eq}}(h)-f_{\mathrm{eq}}\left(h_{0}\right)\right) h^{n_{\mathrm{u}}} P_{j}^{-}\right) \\
& =\frac{1}{\beta} \operatorname{Tr}\left(h^{n_{\mathrm{v}}} P_{j}^{-} \mathrm{i}\left[f_{\mathrm{eq}}(h)-f_{\mathrm{eq}}\left(h_{0}\right), h_{0}^{n_{\mathrm{u}}} 1_{k}\right]\right) .
\end{aligned}
$$

Noticing that

$$
\operatorname{Tr}\left(h_{0}^{n_{\mathrm{v}}} 1_{j} \mathrm{i}\left[f_{\text {eq }}(h)-f_{\text {eq }}\left(h_{0}\right), h_{0}^{n_{\mathrm{u}}} 1_{k}\right]\right)=\operatorname{Tr}\left(\left(f_{\text {eq }}(h)-f_{\text {eq }}\left(h_{0}\right)\right) \mathrm{i}\left[h_{0}^{n_{\mathrm{u}}} 1_{k}, h_{0}^{n_{\mathrm{v}}} 1_{j}\right]\right)=0,
$$

we can rewrite the last identity as

$$
L_{k j}^{\mathrm{uv}}=\frac{1}{\beta} \operatorname{Tr}\left(\left(h^{n_{\mathrm{v}}} P_{j}^{-}-h_{0}^{n_{\mathrm{v}}} 1_{j}\right) \mathrm{i}\left[f_{\mathrm{eq}}(h)-f_{\mathrm{eq}}\left(h_{0}\right), h_{0}^{n_{\mathrm{u}}} 1_{k}\right]\right) .
$$

Since

$$
\left(h^{n_{\mathrm{v}}} P_{j}^{-}-h_{0}^{n_{\mathrm{v}}} 1_{j}\right)\left\langle h_{0}\right\rangle^{-1}=\mathrm{s}-\lim _{t \rightarrow \infty} \int_{0}^{t} \mathrm{e}^{-\mathrm{i} s h} \varphi_{j}^{\mathrm{v}} \mathrm{e}^{\mathrm{i} s h}\left\langle h_{0}\right\rangle^{-1} \mathrm{~d} s,
$$


and $\left\langle h_{0}\right\rangle \mathrm{i}\left[f_{\text {eq }}(h)-f_{\text {eq }}\left(h_{0}\right), h_{0}^{n_{\mathrm{u}}} 1_{k}\right]$ is easily seen to be trace class one has

$$
L_{k j}^{\mathrm{uv}}=\lim _{t \rightarrow \infty} \frac{1}{\beta} \int_{0}^{t} \operatorname{Tr}\left(\mathrm{e}^{-\mathrm{i} s h} \varphi_{j}^{\mathrm{v}} \mathrm{e}^{\mathrm{i} s h} \mathrm{i}\left[f_{\text {eq }}(h)-f_{\text {eq }}\left(h_{0}\right), h_{0}^{n_{\mathrm{u}}} 1_{k}\right]\right) \mathrm{d} s .
$$

Using again Identity (6.56) and a simple $\Lambda$-regularization argument we obtain

$$
L_{k j}^{\mathrm{uv}}=\lim _{t \rightarrow \infty} \frac{1}{\beta} \int_{0}^{t} \mathrm{~d} s \int_{0}^{\beta} \mathrm{d} \theta \operatorname{Tr}\left(\mathrm{e}^{-\mathrm{i} s h} \varphi_{j}^{\mathrm{v}} \mathrm{e}^{\mathrm{i} s h}\left(1-f_{\text {eq }}(h)\right) \mathrm{e}^{-\theta h} \varphi_{k}^{\mathrm{u}} \mathrm{e}^{\theta h} f_{\text {eq }}(h)\right) .
$$

Performing the change of variables $\theta \mapsto \beta-\theta$ in this expression and reorganizing the factors in the trace we can rewrite it as

$$
L_{k j}^{\mathrm{uv}}=\lim _{t \rightarrow \infty} \frac{1}{\beta} \int_{0}^{t} \mathrm{~d} s \int_{0}^{\beta} \mathrm{d} \theta \operatorname{Tr}\left(f_{\text {eq }}(h) \mathrm{e}^{\mathrm{i} s h} \varphi_{k}^{\mathrm{u}} \mathrm{e}^{-\mathrm{i} s h}\left(1-f_{\text {eq }}(h)\right) \mathrm{e}^{-\theta h} \varphi_{j}^{\mathrm{v}} \mathrm{e}^{\theta h}\right) .
$$

Finally we note that for trace class operators $a, b$ on $\mathfrak{h}$ and a gauge-invariant quasi-free state $\omega_{T}$ on $\mathcal{O}$ such that $\omega_{T}(\mathrm{~d} \Gamma(a))=\omega_{T}(\mathrm{~d} \Gamma(b))=0$ it follows from Equ. (2.1) that

$$
\omega_{T}(\mathrm{~d} \Gamma(a) \mathrm{d} \Gamma(b))=\operatorname{Tr}(T a(I-T) b) .
$$

Hence we can rewrite our last formula as

$$
L_{k j}^{\mathrm{uv}}=\lim _{t \rightarrow \infty} \frac{1}{\beta} \int_{0}^{t} \mathrm{~d} s \int_{0}^{\beta} \mathrm{d} \theta \omega_{\text {eq }}\left(\tau^{s}\left(\Phi_{k}^{\mathrm{u}}\right) \tau^{\mathrm{i} \theta}\left(\Phi_{j}^{\mathrm{v}}\right)\right) .
$$

In the time-reversal invariant case one has $J P_{j}^{-} J=P_{j}^{+}=\Omega_{+} 1_{j} \Omega_{+}^{*}$ and $J \varphi_{k}^{\mathrm{u}} J=-\varphi_{k}^{\mathrm{u}}$. These identities and Equ. (6.55) yield

$$
\begin{aligned}
L_{k j}^{\mathrm{uv}} & =\frac{1}{2}\left(L_{k j}^{\mathrm{uv}}+\overline{L_{k j}^{\mathrm{uv}}}\right) \\
& =\frac{1}{2} \operatorname{Tr}\left(\varphi_{k}^{\mathrm{u}}\left(1-f_{\mathrm{eq}}(h)\right)\left(P_{j}^{-}-P_{j}^{+}\right) f_{\mathrm{eq}}(h) h^{n_{\mathrm{v}}}\right) .
\end{aligned}
$$

Since

$$
P_{j}^{-}-P_{j}^{+}=\mathrm{s}-\lim _{t \rightarrow \infty} \int_{-t}^{t} \mathrm{e}^{-\mathrm{i} h s} \varphi_{j}^{\mathrm{c}} \mathrm{e}^{\mathrm{i} s h} \mathrm{~d} s
$$

we obtain

$$
L_{k j}^{\mathrm{uv}}=\lim _{t \rightarrow \infty} \frac{1}{2} \int_{-t}^{t} \operatorname{Tr}\left(\varphi_{k}^{\mathrm{u}}\left(1-f_{\text {eq }}(h)\right) e^{-\mathrm{i} h s} \varphi_{j}^{\mathrm{c}} \mathrm{e}^{\mathrm{i} s h} f_{\text {eq }}(h) h^{n_{\mathrm{v}}}\right) \mathrm{d} s .
$$

If $\mathrm{v}=\mathrm{c}$ then $n_{\mathrm{v}}=0$ and Equ. (6.57) yields

$$
L_{k j}^{\mathrm{uc}}=\lim _{t \rightarrow \infty} \frac{1}{2} \int_{-t}^{t} \omega_{\mathrm{eq}}\left(\tau^{s}\left(\Phi_{k}^{\mathrm{u}}\right) \Phi_{k}^{\mathrm{c}}\right) \mathrm{d} s .
$$


In the case $\mathrm{v}=\mathrm{h}$, we use the identity $\varphi_{j}^{\mathrm{c}} h=\mathrm{i}\left[1_{j}, h\right] h=\mathrm{i}\left[1_{j} h_{0}, h\right]+\mathrm{i}\left[1_{j} v, h\right]=\varphi_{j}^{\mathrm{h}}+\mathrm{i}\left[1_{j} v, h\right]$. Note that

$$
\int_{-t}^{t} \mathrm{e}^{-\mathrm{i} h s} \mathrm{i}\left[1_{j} v, h\right] \mathrm{e}^{\mathrm{i} s h} \mathrm{~d} s=\mathrm{e}^{-\mathrm{i} h t} 1_{j} v \mathrm{e}^{\mathrm{i} t h}-\mathrm{e}^{\mathrm{i} h t} 1_{j} v \mathrm{e}^{-\mathrm{i} t h},
$$

vanishes strongly as $t \rightarrow \infty$. We thus get

$$
L_{k j}^{\mathrm{uh}}=\lim _{t \rightarrow \infty} \frac{1}{2} \int_{-t}^{t} \operatorname{Tr}\left(\varphi_{k}^{\mathrm{u}}\left(1-f_{\mathrm{eq}}(h)\right) e^{-\mathrm{i} h s} \varphi_{j}^{\mathrm{h}} \mathrm{e}^{\mathrm{i} s h} f_{\mathrm{eq}}(h)\right) \mathrm{d} s,
$$

and conclude the proof as in the previous case.

\section{References}

[AE] Anderson, P.W., Engquist, H.-L.: Definition and measurement of the electrical and thermal resistances. Phys. Rev. B 24, 1151 (1981).

[AEG] Avron, J.E., Elgart, A., Graf, G.M., Sadun, L., Schnee, K.: Adiabatic charge pumping in open quantum systems. Commun. Pure Appl. Math. 57, 528 (2004).

[AH] Araki, H., Ho, T.G.: Asymptotic time evolution of a partitioned infinite twosided isotropic $X Y$-chain. Proc. Steklov Inst. Math. 228, 191 (2000).

[AJPP] Aschbacher, W., Jakšić, V., Pautrat, Y., Pillet, C.-A.: Topics in non-equilibrium quantum statistical mechanics. In Open Quantum Systems III. S. Attal, A. Joye, C.-A. Pillet editors. Lecture Notes in Mathematics 1882, Springer, New York (2006).

[AP] Aschbacher, W., Pillet, C.-A.: Non-equilibrium steady states of the $X Y$-chain. J. Stat. Phys. 112, 1153 (2003).

[BP] Ben Saad, R., Pillet, C.-A.: In preparation.

[B1] Büttiker, M.: Four-terminal phase-coherent conductance. Phys. Rev. Lett. 57, 1761 (1986).

[B2] Büttiker, M.: Coherent and sequential tunneling in series barriers. IBM J. Res. Dev. 32, 63 (1988).

[BILP] Büttiker, M., Imry, Y., Landauer, R., Pinhas, S.: Generalized many-channel conductance formula with application to small rings. Phys. Rev. B 31, 6207 (1985).

[BR] Bratteli, O, Robinson D. W.: Operator Algebras and Quantum Statistical Mechanics 2. Second edition, Springer-Verlag, Berlin (1996). 
[CJM] Cornean, H.D., Jensen, A., Moldoveanu, V.: A rigorous proof of the LandauerBüttiker formula. J. Math. Phys. 46, 42106 (2005).

[D] Datta, S.: Electronic Transport in Mesoscopic Systems. Cambridge University Press (1995).

[FL] Fisher, D.S., Lee, P.A.: Relation between conductivity and transmission matrix. Phys. Rev. B 23, 6851 (1981).

[I] Imry, Y.: Introduction to Mesoscopic Physics. Oxford University Press (1997).

[IL] Imry, Y., Landauer, R.: Conductance viewed as transmission. Rev. Mod. Phys. 71, S306 (1999).

[IWP] Inagaki, M., Wanders, G., Piron C.: Théorème $H$ et unitarité de $S$. Helv. Phys. Acta 27, 71 (1954).

[JL1] Jakšić, V., Last, Y.: Spectral structure of Anderson type Hamiltonians. Invent. Math. 141, 561 (2000).

[JL2] Jakšić, V., Last, Y.: Simplicity of singular spectrum in Anderson type Hamiltonians. Duke Math. J. 133, 185 (2006).

[JP1] Jakšić, V., Pillet, C.-A.: Non-equilibrium steady states of finite quantum systems coupled to thermal reservoirs. Commun. Math. Phys. 226, 131 (2002).

[JP2] Jakšić, V., Pillet, C.-A.: Mathematical theory of non-equilibrium quantum statistical mechanics. J. Stat. Phys. 108, 787 (2002).

[JKP] Jakšić, V., Kritchevski, E., Pillet, C.-A.: Mathematical theory of the WignerWeisskopf atom. In Large Coulomb Systems. Lecture Notes on Mathematical Aspects of QED. J. Derezinski, H. Siedentop editors. Lecture Notes in Physics 695, Springer, New York (2006).

[J0P1] Jakšić, V., Ogata, Y., Pillet, C.-A.: The Green-Kubo formula and the Onsager reciprocity relations in quantum statistical mechanics. Commun. Math. Phys. 265, 721 (2006).

[J0P2] Jakšić, V., Ogata, Y., Pillet, C.-A.: Linear response theory for thermally driven quantum open systems. J. Stat. Phys. 123, 547 (2006).

[J0P3] Jakšić, V., Ogata, Y., Pillet, C.-A.: The Green-Kubo formula for the spinfermion system. To appear in Commun. Math. Phys. (2006).

[J0P4] Jakšić, V., Ogata, Y., Pillet, C.-A.: The Green-Kubo formula for locally interacting fermionic open systems. Preprint (2006). 
[L] Landauer, R.: Electrical resistance of disordered one-dimensional lattices. Phil. Mag. 21, 863 (1970).

[LA] Langreth, D.C., Abrahams, E.: Derivation of the Landauer conductance formula. Phys. Rev. B 24, 2978 (1981).

[MW] Meir, Y., Wingreen, N.S.: Landauer Formula for the current through an interacting region. Phys. Rev. Lett. 68, 2512 (1992).

[Ro] Robinson, D.W.: Return to equilibrium. Commun. Math. Phys. 31, 171 (1973).

[Ru1] Ruelle, D.: Natural nonequilibrium states in quantum statistical mechanics. J. Stat. Phys. 98, 57 (2000).

[Ru2] Ruelle, D.: Entropy production in quantum spin systems. Commun. Math. Phys. 224, 3 (2001).

[S] Stückelberg, E.C.G.: Théorème $H$ et unitarité de $S$. Helv. Phys. Acta 25, 577 (1952).

[SI] Sivan, U., Imry, Y.: Multichannel Landauer formula for the thermoelectric transport with application to thermopower near the mobility edge. Phys. Rev. B 33, 551 (1986).

[T] Thouless, D.J.: Why Landauer's formula for resistance is right. Phys. Rev. Lett. 47, 972 (1981).

[TT1] Takahashi, J., Tasaki, S.: Nonlinear transport and current fluctuation in AB Ring with a quantum dot. J. Phys. Soc. Jpn. 74, Suppl. 261 (2005).

[TT2] Takahashi, J., Tasaki, S.: Nonequilibrium steady states and MacLennanZubarev ensembles in a quantum junction system. Preprint cond-mat/0606259 (2006).

[TT3] Takahashi, J., Tasaki, S.: Nonequilibrium staedy states and Fano-Kondo resonances in an AB ring with a quantum dot. Preprint cond-mat/0603337 (2006).

[Y] Yafaev, D.R.: Mathematical Scattering Theory: General Theory. AMS, Providence, Rhode Island (1992). 